\title{
Optical Injection Locking: from Principle to Applications
}

\author{
Zhixin Liu, Senior Member, IEEE, Radan Slavík, Senior Member, IEEE
}

(Invited Tutorial)

\begin{abstract}
This paper reviews optical injection locking (OIL) of semiconductor lasers and its application in optical communications and signal processing. Despite complex OIL dynamics, we attempt to explain the operational principle and main features of the OIL in an intuitive way, aiming at a wide understanding of the OIL and its associated techniques in the optic and photonic communities. We review and compare different control techniques that enable robust OIL in practical systems. The applications are reviewed with a focus on new developments in the past decade, under the categories of 'High Speed Directly Modulated Lasers' and 'Optical Carrier Recovery'. Finally, we draw our vision for future research directions.
\end{abstract}

Index Terms-Laser dynamics, Optical communications, Optical transmitters, Optical injection locking, Direct modulation, Coherent receiver, Carrier recovery, Frequency comb, Time and frequency transfer

\section{INTRODUCTION}

$\mathbf{O}$ PTICAL injection locking (OIL) is an optical frequency and phase synchronization technique based on the photon-photon interaction when an external light is shined into a laser cavity [1]. Fig.1 shows two typical OIL experimental setups. The OIL system contains a 'master' laser that provides the external seeding light to influence a 'slave' laser. The master light is injected into the slave laser either via a circulator (reflection type, Fig.1a) or an isolator (transmission type, Fig.1b) to avoid any parasitic reflection back to the master laser. When the master laser carrier frequency (wavelength) is close enough to the free-running lasing carrier frequency (wavelength) of the slave laser, the slave laser will be forced to synchronize with the master laser, i.e. lasing at the same frequency with a fixed phase offset. The slave

This Manuscript received xxx, 2019; revised xxx; accepted xxx. Date of publication xxx; date of current version xxx. This work was supported by the UK Engineering and Physical Sciences Research Council under EP/R041792/1, EP/R035342/1(TRANSENT), and Royal Society Paul Instrument Fund (PIF/R1/180001).

Z. Liu is with the Department of Electronic and Electrical Engineering at University College London, London, WC1E 7JE, U.K. (e-mail: zhixin.liu@ucl.ac.uk)

R. Slavík is with the Optoelectronics Research Centre, University of Southampton, Southampton SO17 1BJ, U.K. (e-mail: R.Slavik@soton.ac.uk).
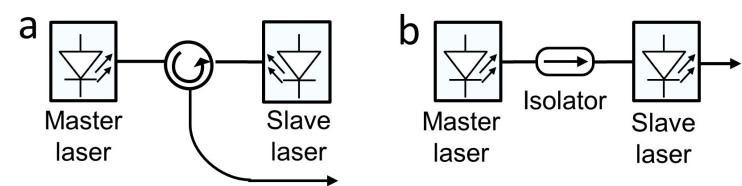

Figure 1. Schematics of optical injection-locked laser system. (a) Reflection type and (b) Transmission type.

laser will also follow any slow frequency drift of the master laser with a relatively constant output power.

Laser synchronization plays important roles in numerous applications. In optical communications, synchronized lasers are used as local oscillators (LOs) to reduce the complexity and latency in coherent receivers [2]-[5]. In conjunction with optical frequency comb, OIL coherently demultiplexes optical tones for dense wavelength division multiplexed (DWDM) systems or super channel transmitters [6]-[9], enabling high spectral efficiency communications. In sensing and quantum communications, OIL provides high sensitivity detection of phase modulated signals [10]. In optical science and instrumentation, phase synchronized lasers are coherently combined to generate arbitrary shaped optical pulses [11], [12]. Similar techniques have also been used for microwave signal generations for beam-forming networks in phased-array antennas [13].

Laser synchronization can be achieved with other techniques than OIL. For example, optical phase-lock loops (OPLLs) use purely electronics feedback to control the slave laser carrier frequency (e.g., by controlling its bias current). However, OPLLs require fast electronics with typically 100 times higher loop bandwidth than the linewidth of the free-running slave laser. In contrast, OIL allows the slave laser to lock with the master laser over large bandwidth (10s of $\mathrm{GHz}$ ) using slow control electronics (typically $10 \mathrm{kHz}-10 \mathrm{MHz}$ ), significantly reducing the complexity, cost, and power consumption of laser locking electronics. The large locking bandwidth also brings the merits of improved phase and intensity noise performance when a high quality (low noise) master laser is used. 
Apart from frequency and phase synchronization, OIL was demonstrated to significantly enhance the modulation bandwidth of directly modulated slave lasers (DMLs) with a simultaneous reduction of the unwanted modulation chirp [14]-[16] and relative intensity noise (RIN) [17], [18]. These attractive features have raised long-lasting research interest in injection-locked DMLs (OIL-DML) in the last four decades. With the added beneficial features like reduced threshold bias current [19] and improved modulation linearity [20], it has received attention in applications not only within optical communications, but also in analog photonics including radars and telescopes.

Tracing back the history, scientists started research on the OIL phenomenon since the invention of lasers [21]. For over half a century, researchers have made numerous efforts on the theoretical understanding of the OIL phenomenon and the experimental development of relevant technologies, yet there are still new scientific findings and increasingly strong interests in application of OIL technologies. Especially in the last decade, we have witnessed practical applications of OIL in numerous important fields, which gave us the motivation to write this tutorial paper.

This paper is organized as sketched in Fig. 2. We first review the history and attempt to convey an intuitive understanding of the operational principle of OIL in Section II. The key parameters are summarized to enable semi-analytical understanding of the OIL features to be discussed in Section III. Next, in Section IV, we discuss three control techniques and their corresponding conditions for stable OIL. Although not discussed in most scientific papers, the control methods are crucial to make the OIL techniques practical. Subsequently, we discuss the application of OIL in high speed DML in Section V. Section VI reviews the OIL-based optical carrier recovery and its relevant applications. The review of applications focuses on the progress in the last decade, aiming to link the great history of OIL with the current research progress. A summary of the paper with our visions on future research and development is presented in Section VII before the conclusion in Section VIII. We limit our discussions to OIL operating in the stable locking regime with the non-stable OIL dynamics such as four-wave mixing (FWM) [22], laser pulling and chaos effects [23] outside the scope of this paper.

The core of this paper rises from our experimentalist souls, which later became convinced of the importance of theory. For researchers new to the term "optical injection locking", we suggest starting from the Section II. For readers already familiar with OIL but wishing to grasp a summary of recent advance, we recommend going

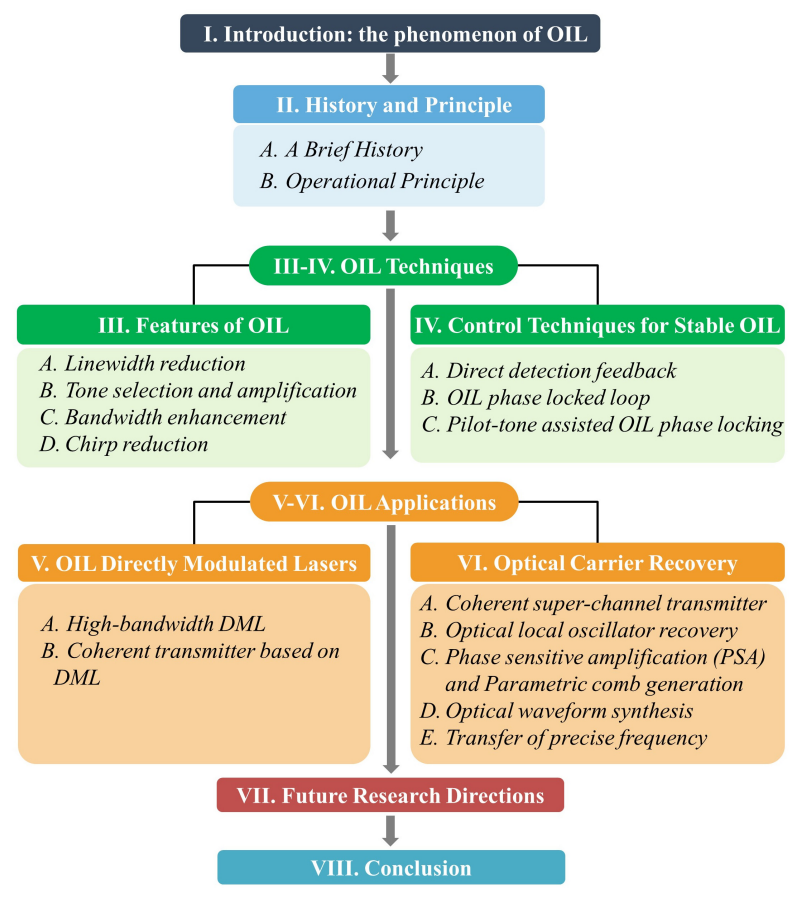

Figure 2. Structure of this tutorial.

directly to the Section IV.

\section{History AND PRINCIPLE}

\section{A. A Brief History}

The first observation of the injection locking effect was perhaps the synchronization of pendulum clocks. In 1665, the Dutch physicist Christian Huygens, who invented the pendulum clock, observed that no matter what initial phase the pendulums were started, they always synchronized with each other within a short period of time. This is because as the pendulums move back and forth, sound pulses travel through the wall and create vibrations that interfere with the pendulum swing [24]. Evolving from mechanics to electronics, the injection locking of electronic oscillators was first reported in 1919, in which an electronic oscillator was disturbed and synchronized to a weak oscillator input connected in a feedback circuit [25]. This oscillator synchronization technique soon stimulated interests in the 'new field' of electronic engineering and found a number of applications in electronic devices and communications, such as frequency dividers, quadrature amplitude modulation, and oscillators locked with fine phase separation. A milestone of oscillator injection locking theory was marked by Alder, who derived the seminal differential equation that described the fundamental dynamics of oscillator 
injection locking in his 1946 paper [26]. This theory was later extended by Paciorek by considering strong signal injection, forming the general theoretical ground for oscillator injection locking [27].

With the birth of lasers in 1960 [28], the concept of injection locking was transferred to the field of optics. Stover and Steier reported the first OIL experiment in 1966 by using two He-Ne lasers [21]. In the same period, the classical theory was extended to include laser locking, predicting symmetric locking range in gas and dye lasers [29], [30].

The invention of the semiconductor laser and optical fiber opened the door for a range of technologies that form the modern information and communication infrastructure. The concepts of frequency and phase modulation/demodulation in classic radio frequency (RF) communications was brought into the field of optical communications. The phase synchronized local oscillator (LO) plays an important role in the demodulation of phase modulated signals. Motivated by this need, OIL of a semiconductor laser was experimentally studied [31], followed by a modification of the classic theory by Lang, who incorporated the linewidth enhancement factor into the OIL laser rate equation [32]. This modelling is now widely accepted as a standard way of analyzing the OIL dynamics in semiconductor lasers.

Following the maturation of semiconductor laser manufacturing, researchers started an active period of exploring the application of OIL in optical communication systems in the 1980s. One representative example is to suppress the modulation chirp using OIL to extend reach of an intensity modulated direct detection (IM-DD) system, in which the modulation chirp combined with the fiber chromatic dispersion limit the reach [33]. Other examples include generating phase modulated signals using injection locked lasers [34], and OIL based LO recovery for coherent homodyne detection [2], [35].

In the 1990s, the invention of erbium-doped fiber amplifier (EDFA) and dispersion compensation fiber (DCF) significantly changed the paradigm of optical communications. The OIL technology that benefited coherent detection and reduced power penalty from chromatic dispersion was not attractive during this period, resulting in a fall of research interest on this topic. Nevertheless, researchers made consistent efforts advancing the theory and applications, such as the research on strong OIL [36], [37] and the demonstration of OIL phase lock loops [38].

Entering the 2000s, the need for high-bandwidth DML for radio-frequency (RF) applications and high-speed telecommunications stimulated intense interests in the OIL-DMLs. More than ten times bandwidth enhance- ment was demonstrated using strong OIL to standard distributed feedback (DFB) lasers and vertical cavity surface emitting lasers (VCSEL) [39], [40]. In the meantime, researchers explained the origin of bandwidth enhancement with further improvement of the theoretical modelling [41], [42]. A tutorial paper by Lau reviewed the development of high-speed OIL-DMLs during this period [43].

In the last decade, we have witnessed a strong interest in OIL for telecom, microwave, and instrument applications with successful commercialization. The need for laser synchronization has made OIL a fundamental building block for various applications, together with a significant increase in the number of publications explicitly focusing on system applications. Standing at this historical point of OIL development, we hope to capture the key advances and remaining challenges with a systematic review of OIL and the relevant technologies.

\section{B. Operational principle}

In contrast to pendulums in which sound waves travel in both ways, optical injection locking refers to one laser affecting the other, as described in Fig.1. Note that this is different from two-section lasers in which light from both lasers affects each other [44]. The widely accepted modelling of OIL dynamics is a modification of a semiclassical laser rate equations by introducing additional terms of light intensity (or the number of photons) and phase offset to describe the impact from the master laser signal (e.g., [19], [41], [43]). From [41]:

$$
\begin{aligned}
& \frac{d A(t)}{d t}=\frac{1}{2} g\left[N(t)-N_{t h}\right] A(t)+\kappa A_{i n j} \cos \phi(t) \\
& \frac{d \phi(t)}{d t}=\frac{\alpha}{2}\left\{g\left[N(t)-N_{t h}\right]\right\}-\kappa \frac{A_{i n j}}{A(t)} \sin \phi(t)-\Delta \omega
\end{aligned}
$$

$$
\frac{d N(t)}{d t}=J-\gamma_{N} N(t)-\left\{\gamma_{p}+g\left[N(t)-N_{t h}\right]\right\} A(t)^{2}
$$

where $A(t)$ is the field amplitude normalized as $A^{2}(t)=S(t)$, and $S(t)$ is the photon number. $\phi(t)$ is the phase difference between the internal and injected field, expressed by $\phi(t)=\varphi_{s}(t)-\varphi_{i n j}(t)$, where $\varphi_{s}(t)$ and $\varphi_{i n j}(t)$ are the phases of the slave and the master lasers, respectively. $\Delta \omega=\omega_{i n j}-\omega_{s}$ is the angular frequency offset between the the free-running slave lasers $\left(\omega_{s}\right)$ and the injected light $\left(\omega_{i n j}\right)$. Equation (3) governs the carrier change which is the same as the standard rate equation, where $N(t)$ is the number of carriers in the slave laser. 
Table I

INJECTION-LOCKED LASER PARAMETERS

\begin{tabular}{cl}
\hline Symbol & Parameters \\
\hline $\mathrm{g}$ & laser gain coefficient \\
$N_{t h}$ & threshold carrier number \\
$N_{t r}$ & transparency carrier number \\
$\kappa$ & coupling coefficient \\
$A_{i n j}$ & Field amplitude of the injected light \\
$\Delta \omega$ & frequency offset between the master and \\
$\alpha$ & free running slave $\omega_{i n j}-\omega_{s}$ \\
$\gamma_{N}$ & linewidth enhancement factor \\
$\gamma_{p}$ & carrier recombination rate \\
$J$ & photon decay rate \\
& charge $e$ \\
\hline
\end{tabular}

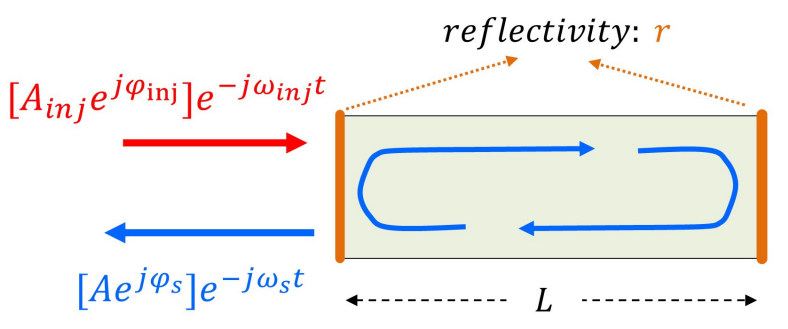

Figure 3. Slave laser parameters including input and output light fields used in modelling of the OIL dynamics.

Other rate equation parameters are summarized in Table I. In this paper, $\omega$ always refers to angular frequency $\omega=2 \pi f$. Thus, $e^{j \omega t}$ is the time oscillation factor. The optical field of the free running slave laser is represented by $\left[A e^{j \varphi_{s}}\right] e^{-j \omega_{s} t}$.

Compared to the free-running laser rate equations, OIL rate equations have additional terms in (1) and (2), as highlighted in red. As shown in Fig. 3, master light $\left[A_{i n j} e^{j \varphi_{i n j}}\right] e^{-j \omega_{i n j} t}$ injects into the slave laser, where $A_{i n j} e^{j \varphi_{i n j}}$ is the complex field of the injected light. $\kappa$ is the coupling coefficient that describes the rate at which the injected photons enter into the cavity [42]:

$$
\kappa=\frac{C}{2 n_{g} L} \frac{1-r}{\sqrt{r}}=\frac{\omega_{s}}{2 Q}
$$

where $n_{g}$ is the group refractive index of the laser waveguide, $L$ is the cavity length, $r$ is power reflectivity of the cavity mirrors, and $Q$ is the cavity quality factor.

Following the steady state solutions derived in [41], we can obtain stable OIL (where slave laser carrier frequency tracks that of the master laser) provided $\Delta \omega=\omega_{i n j}-\omega_{s}$ is within certain bounds, $\Delta \omega_{\min }$ to $\Delta \omega_{\max }$. The locking range is then

$$
\Delta \omega_{L R}=\Delta \omega_{\max }-\Delta \omega_{\min }
$$

In literature $\Delta \omega_{L R}$ is sometimes referred to as 'locking bandwidth'. It can be obtained by considering the steady state solution of the OIL rate equations (1)-(3), which gives [43]:

$-\kappa \sqrt{1+\alpha^{2}} \sqrt{\frac{P_{i n j}}{P_{o}}}=\Delta \omega_{\min }<\Delta \omega<\Delta \omega_{\max }=\kappa \sqrt{\frac{P_{i n j}}{P_{o}}}$

where $P_{o}$ and $P_{i n j}$ are the output power of the slave lasers and the power of the injected signal, respectively. From (6) we see that locking range is given by: 1) coupling coefficient $\kappa, 2$ ) linewidth enhancement factor $\alpha$, and 3) injection ratio defined as $\frac{P_{i n j}}{P_{o}}$.

Since the coupling coefficient $\kappa$ is inversely proportional to the quality factor $Q$, a slave laser with low $Q$ will have a larger locking range than lasers with high $Q$. Therefore, low $Q$ lasers are easier to lock. Considering an extreme case where $Q$ tends to zero, the slave laser degenerates to a gain medium (e.g. a semiconductor optical amplifier (SOA)) that amplifies any injected signal within the gain region. However, low $Q$ also leads to increased laser linewidth that reduces the phase noise performance of the OIL laser system.

A necessary condition for stable OIL is the equation (2) has a steady state solution. Physically, it means the slave laser must reduces its gain (or carrier number) to achieve a steady state (i.e. $\phi$ is a constant value) that locks to the master laser. Due to the nonzero linewidth enhancement factor $\alpha$, which describes the amplitude phase coupling (i.e. phase change due to the change of carrier density dependent refractive index), the cavity mode is red shifted (negative frequency detuning), which causes the asymmetry of the locking range. Should the $\alpha$ becomes zero, the equation (2) degenerates to an equation independent of carrier number $N$, and will result in a symmetric locking range. Recent advance in quantum dot lasers shows that low or zero $\alpha$ can be achieved [45], providing new opportunities to the field of OIL dynamics.

For a given laser, the locking range is proportional to the square root of the injection ratio $\frac{P_{i n j}}{P_{o}}(6)$. Fig.4 plots the OIL slave laser status under different injection ratios and frequency offsets. The shaded area shows the stable locking region. Generally speaking, high injection ratio results in a large locking range, which also makes the slave laser easier to lock. Using the example parameters listed in Fig.4, we calculated the locking range at -20 $\mathrm{dB}$ injection ratio to be about $12 \mathrm{GHz}$. The frequency detuning between the master and slave lasers $\Delta f$ ranges from -9 to $3 \mathrm{GHz}$ without losing OIL. Commercial lasers with thermoelectric cooling (TEC) can in laboratory conditions keep drift well below $12 \mathrm{GHz}$ and thus 
can remain OIL locked for extended period of time (e.g., hours). However, when the injection ratio reduces, the locking range significantly decreases, e.g., in our example shown in Fig.4 it reaches $370 \mathrm{MHz}$ for injection ratio of $-50 \mathrm{~dB}$. As a result, the OIL lock can be lost quickly (after seconds/minutes) due to the drifts in the slave or master lasers bias currents or temperatures. This necessities additional feedback control to keep stable OIL (discussed in Section IV).

Under stable OIL, the steady state phase of the slave laser is determined by the frequency offset and locking range [19], [43]:

$$
\begin{aligned}
\phi & =\sin ^{-1}\left\{-\frac{\Delta \omega}{\kappa \sqrt{1+\alpha^{2}}} \sqrt{\frac{P_{o}}{P_{\text {inj }}}}\right\}-\tan ^{-1} \alpha \\
& =\sin ^{-1}\left\{\frac{\Delta \omega}{\Delta \omega_{\min }}\right\}-\tan ^{-1} \alpha
\end{aligned}
$$

Thus, although the slave laser is frequency locked to the master, their relative phase difference (phase offset) may not be constant, but stays within the region of $\cot ^{-1} \alpha$ to $-\pi / 2$, as shown in Fig. 4. Within the OIL region, any variation of frequency detuning $\Delta \omega$ is converted into the phase variation of the slave laser output. In this sense, the OIL system acts as an optical frequency discriminator. The range of this 'frequencyphase conversion' depends on $\kappa$ (7) (and thus also on the slave laser $Q$ factor (4)). This conclusion also agrees with the classic injection locking theory derived for electronic oscillator [26].

\section{Features of Optical InJection Locking}

\section{A. Linewidth reduction}

Narrow linewidth lasers are crucial for applications including coherent optical communication systems and microwave photonics. Their linewidth is normally reduced via extending photon life time using external cavity or by locking the laser to a stable reference with advanced electronics [46], [47]. Both these approaches unavoidably add cost that is at odds with the need for low cost narrow linewidth lasers, creating a long-lasting dilemma in practical system applications.

Using OIL, low cost lasers with relatively large linewidth can be turned into high performance narrow linewidth lasers. Fig.5 shows an experimental example we carried out using a $5 \mathrm{kHz}$ fiber laser master to OIL a $1.2 \mathrm{MHz}$ linewidth discrete mode semiconductor slave laser [48]. The laser linewidth was measured using a selfheterodyne setup with an $80 \mathrm{~km}$ standard single mode fiber (SMF-28) delay line [49]. The $80 \mathrm{~km}$ of delay in an optical fiber corresponds to $80 \times 1.45=120 \mathrm{~km}$ in free space (1.45 is the fiber refractive index). Given
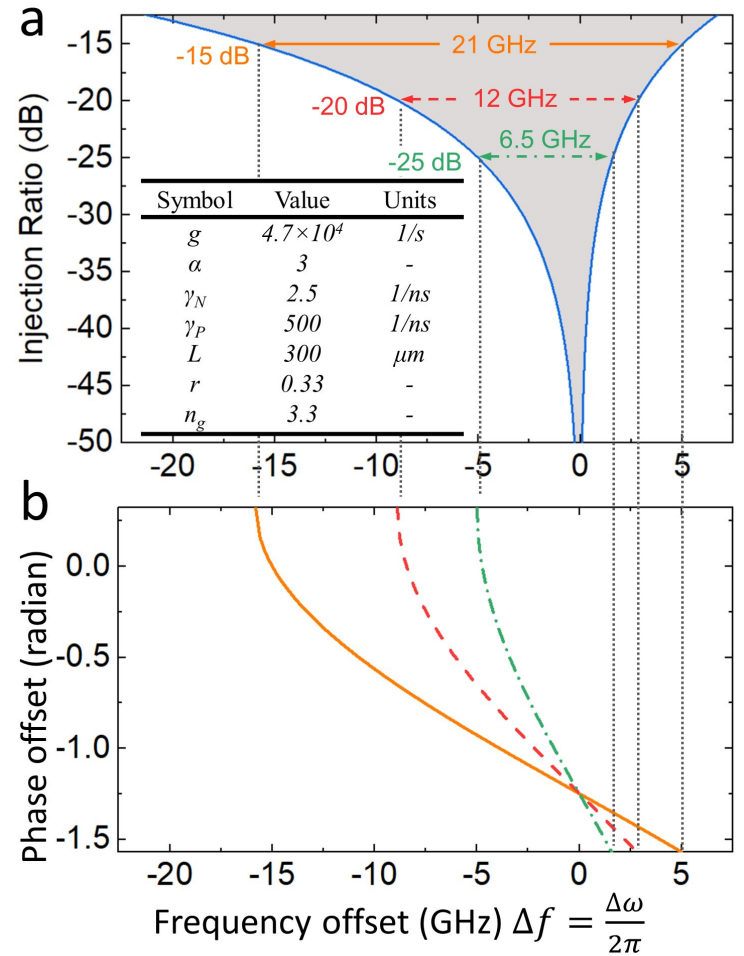

Figure 4. (a) Locking range as a function of the injection ratio and (b) Phase offset between the master and the OIL slave lasers as a function of the master-free running slave lasers frequency offset for three selected injection ratios.

that Coherence length $=c /(\pi \times$ linewidth $), 120 \mathrm{~km}$ allows for measurement of linewidths down to $1 \mathrm{kHz}$ level. However, for a reliable measurement, 3 times the coherence length is usually required, allowing for $i 3 \mathrm{kHz}$ linewidth to be measured in our set-up. As shown in Fig. 5 , even at a low injection ratio of $-40 \mathrm{~dB}$, the linewidth of the slave laser was significantly reduced to about 5 $\mathrm{kHz}$ after OIL. Stable OIL with further power reduction is possible only when appropriate electronic control is applied to ensure $\Delta \omega$ stays within the locking range, as the locking range becomes too narrow to accommodate for the master/slave laser drifts. With the pilot assisted electronic control (to be discussed in Section IV), stable OIL was demonstrated with injection ratio as low as -65 $\mathrm{dB}$ with a $250 \mathrm{MHz}$ pilot [50]. A further reduction of injection ratio to $-75 \mathrm{~dB}$ was achieved in [51] by using $10 \mathrm{GHz}$ pilot tones. Given how little power is needed for OIL, it is possible to split the output of a narrow linewidth master laser to injection lock many low cost slave lasers, enabling a large number of low-cost, narrow linewidth lasers. 

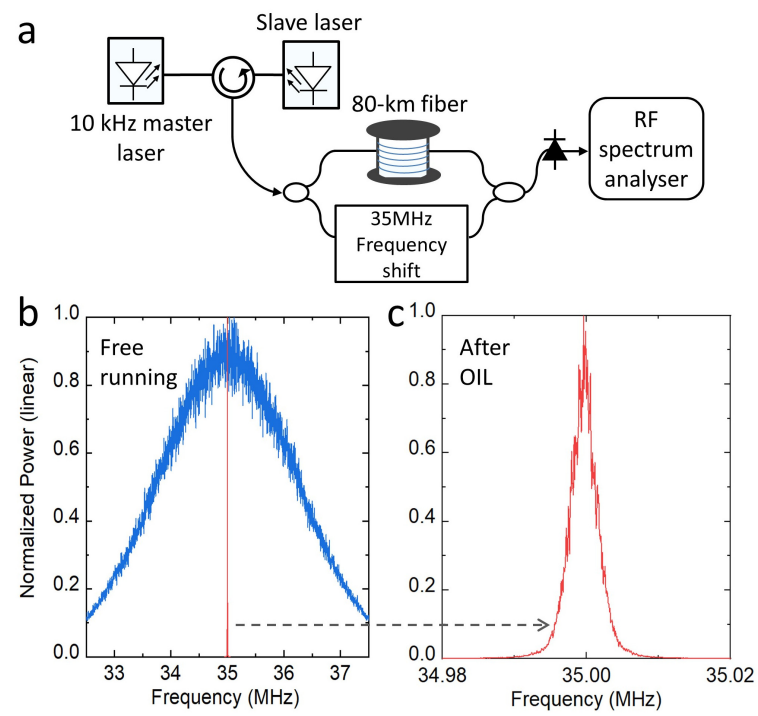

Figure 5. (a) Schematics of self-heterodyne measurement of the slave laser linewidth with (b) the self-heterodyne beat of a free running (blue) and OIL (red) slave laser. (c) The beat of OIL slave laser shown over significantly narrower bandwidth. Linewidth was reduced from $1 \mathrm{MHz}$ to $5 \mathrm{kHz}$.

\section{B. Tone selection and amplification}

In DWDM optical communications [52], optical arbitrary waveform generation [11], [53], [54], and microwave photonics [55], one often needs to isolate and amplify a single-frequency tone signal. Conventionally, this is realized using optical filters, for example, arrayed waveguide gratings (AWG) or wavelength selective switches (WSS), which usually introduce an insertions loss of 3-5 dB. The demultiplexed tone is subsequently amplified via an EDFA or SOA to boost the power. This 'filter + amplifier' scheme unavoidably reduces optical signal-to-noise ratio (OSNR) and can occupy considerable space even on an integrated photonic chips. An alternative method that provides simultaneously low noise and high gain filtering is OIL. When seeding a single-wavelength slave laser (e.g. distributed Bragg reflector laser (DFB) or discrete mode laser) with multiwavelength signals, the carrier frequency of the slave laser can be tuned to the wavelength of the signal to be demultiplexed/extracted. When the slave laser OIL to the selected input channel, it performs optical filtering by amplifying the selected channel (i.e., signal within its locking range), but attenuating the out-of-locking range signal (other channels). The simultaneous demultiplexing and amplification of individual wavelength signals/tones [11] makes OIL an ideal technique for applications requiring tone selection (e.g., from an optical frequency

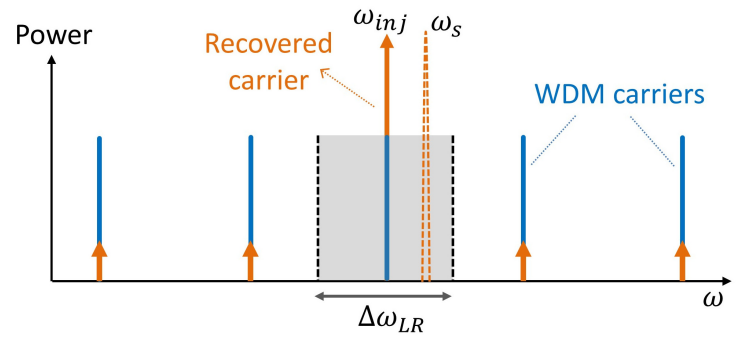

Figure 6. A slave laser can be OIL to a single mode of an Optical Frequency Comb (OFC) if the locking range is smaller than the comb tones spacing. The slave laser will emit at the same frequency as the comb tone to which it is locked to.

comb), as schematically shown in Fig.6.

Compared to the "filter + amplifier" method, OIL provides high gain (by up to $70 \mathrm{~dB}$ [51]) with low added intensity noise. The regenerated tone, however, may pick up additional phase noise due to the variation of the frequency offset between the master and slave lasers (described by (7)). Fortunately, this added phase noise can be suppressed using one of the techniques described in Section IV. It is worth noting that the OIL filtering effect is different in transmission and reflection type OILs. In reflection type configuration, a significant amount of the injected light is reflected by the front facet, which includes the channels/signals to be rejected. This unwanted effect can be reduced by lowering the injection ratio (e.g. via attenuation of the master light). Significantly better performance is obtained when using the rear-facet (transmission) OIL. In this configuration, the unwanted signals/tone channels are reflected back to the master, being strongly-suppressed in the transmission, where the main slave laser output is. The features of high gain and low noise tone selection are particularly desirable for low power multi-tone light sources such as integrated microring resonator frequency combs and mode locked lasers [56], [57]. Its application in conjunction with frequency combs received significant interest in the past decade. We will review this later in Section VI-A.

\section{Bandwidth enhancement}

In the previous section, we discussed OIL with a low injection ratio, i.e. a relatively weak master signal was injected into the slave laser. Here, we will discuss one of the most interesting OIL features that occurs when the injected master signal is relatively strong - strong enough to modify the slave laser dynamics and thus also its response to its bias current modulation. 
The modulation bandwidth of a solitary semiconductor laser (e.g. Fabry-Perot (FP) or DFB laser) is approximated by [58]:

$$
f_{3 d B} \approx\left(\frac{3}{4 \pi^{2} q} \frac{\Gamma \nu_{g} \sigma_{g}}{V}\left(I_{b}-I_{t h}\right)\right)
$$

where $\mathrm{q}$ is the elementary charge, $\Gamma$ is the confinement factor, $\nu_{g}$ is the light group velocity in the laser cavity, $\sigma_{g}$ is the differential gain, $\mathrm{V}$ is the active volume, $I_{b}$ the bias current and $I_{t h}$ the threshold current. Equation (8) indicates that we can increase modulation bandwidth by having short cavity length and/or a high bias current that provides high optical power. Nevertheless, the short cavity results in small power that goes against the need for high power. Further, high bias current unavoidably leads to excess heating and potential facet damage.

OIL was found to bypass these trade-offs, enabling modulation bandwidth enhancement [59]. The physical insight into this phenomena was subjected to debate over more than two decades and was convincingly explained by Murakami in 2003 [41]. From a practical point of view, the externally injected light reduces the carrier number by $\Delta N=N-N_{t h}$, which leads to a red shift of the cavity resonance frequency of the slave laser (from $\omega_{0}$ to $\left.\omega_{0}+\frac{\alpha}{2} g \Delta N\right)$. Consequently, the angular frequency offset between the cavity resonance frequency $\omega_{\text {cav }}$ and the emitting frequency (or the injection frequency $\omega_{i n j}$ when the laser is locked) becomes $\omega_{\text {res }}=\Delta \omega-\frac{\alpha}{2} g \Delta N$, as shown in Fig.7b. This resonance frequency increases with positive frequency detuning, creating a beat between the resonance field and the laser frequency (known as photon-photon resonance) shown as the peak around $40 \mathrm{GHz}$ in Fig.7a. Changing the frequency detuning $\Delta \omega$ also results in a change of the damping factor that leads to a more flat response with less bandwidth enhancement, as shown by the red and blue curves in Fig.7a. In principle, a resonance peak of $>100 \mathrm{GHz}$ frequency is possible provided the injection ratio is strong enough ( $>10 \mathrm{~dB})$. Lau [43] numerically solved the OIL rate equations (1)-(2) and provided a series of locking maps that illustrate the influence of various laser parameters.

\section{Chirp reduction}

Modulation bandwidth discussed in the previous section determines how much information we can modulate on the DML slave laser. Another important parameter is the reach (how far we can transmit it). Due to the chromatic dispersion of optical fibers, the reach is compromised when the modulated signal is chirped. Unfortunately, the intensity modulation of a semiconductor
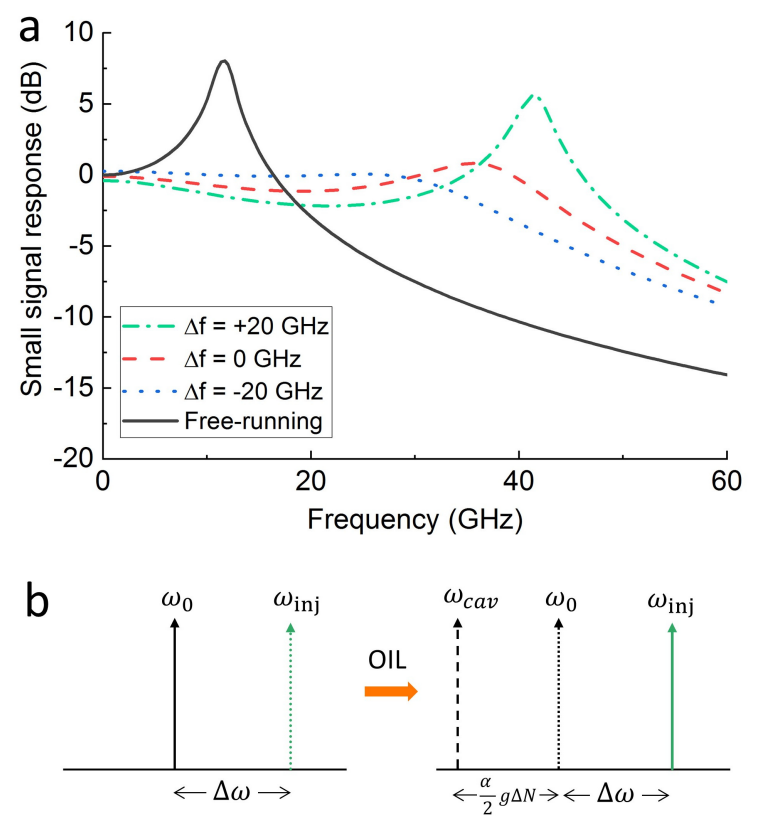

Figure 7. (a) Example of the modulation response of an OIL laser. Assuming OIL ratio of $0 \mathrm{~dB}$, the small signal responses of the OIL laser with different frequency offset OIL ( $\Delta \mathrm{f}=-20$ (dotted blue), 0 (red dashed line), $20 \mathrm{GHz}$ (green dot-dashed line) are shown in the figure. (b) Change of resonance frequency due to injection locking using positive frequency detuning as example. After OIL, the slave laser outputs at $\omega_{i n j}$, the resonance frequency red shifts to $\omega_{c a v}$.

laser is accompanied by the frequency chirp, which is governed by [58]:

$$
\delta \nu(t)=\frac{1}{2 \pi} \frac{d \phi}{d t}=\frac{\alpha}{4 \pi}\left[g\left(N-N_{t r}\right)-\gamma_{p}\right]
$$

For an intensity modulated optical signal generated by a semiconductor DML, the frequency chirp is positive at the leading edge (blue shift) and negative (red shift) towards the trailing edge, resulting in a considerably broadened optical spectrum. OIL has been found to significantly reduce the chirp effect when the slave laser operates in the stable locking region [15], [16]. To gain an intuitive understanding of this, let us first consider a slave laser locked at the center of the locking range, as shown in Fig. 4. When modulating the slave laser, its free running frequency deviates from the center of the locking region due to the frequency chirp. Under the OIL, its frequency stays constant (following the CW master). Although there is still some residual level of the modulation chirp (as the slave laser chirp is converted to phase variation due to the frequency discrimination effect as shown in Fig.4b), the modulation frequency chirp is strongly reduced. The level of the chirp suppression depends on the injection ratio, frequency detuning (used, 

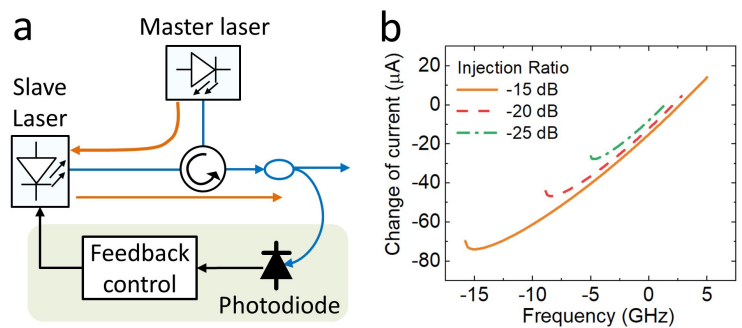

Figure 8. (a) Direct detection based OIL feedback control. (b) Change of photo current (detected by the photodiode in (a) within the locking range.

e.g., to increase modulation bandwidth), and the level of the slave laser current modulation.

The above description aims to intuitively explain the phenomena with rigorous description of the laser dynamics (using the nonlinear laser rate equation) found, e.g., in [15], [16].

\section{Control Techniques for Stable OIL}

In practical systems, the random drifts of temperature and laser bias currents can easily cause master-slave frequency detuning $\Delta \omega$ drifts outside the locking range $\Delta \omega_{L R}$, causing unlocking of the OIL laser. Thus, the frequency offset needs to be controlled. Fine control of $\Delta \omega$ is particularly important for applications requiring low injection ratio because of the small locking range. Such control may also be required even when the injection ratio is relatively large, as variations in $\Delta \omega$ cause undesirable variations in the relative phase between the master and the slave lasers $\phi$ (equation (7)), e.g. for coherent signal synthesis that we will discuss later in Section V-B. In this section, we review three control methods for OIL.

\section{A. Direct detection feedback}

For OIL systems with a modest-to-high injection ratio (e.g., OIL-enhanced directly modulated lasers, OIL for modulation chirp suppression, etc.), the direct detection based feedback control provides a simple and low cost solution, as schematically described in Fig. 8.

The principle is based on the fact that the slave output power $P_{o}$ increases with positive frequency detuning $\Delta \omega$. This phenomenon is well explained in [43]. An example of slave output power as a function of $\Delta \omega$ for three different injection ratios is shown in Fig. 8b. We see that for low injection ratio the signal is getting weaker (change of current is smaller), making this technique less suitable for very low OIL ratios.

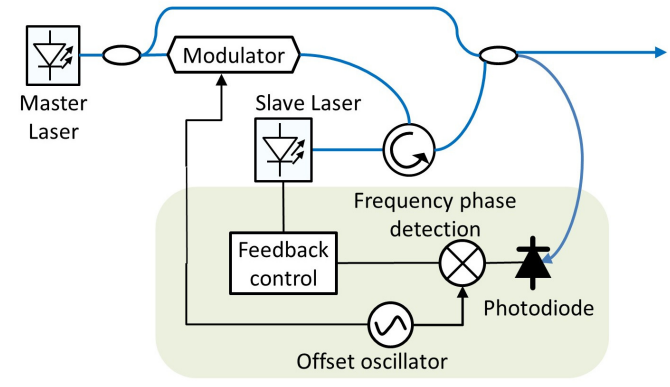

Figure 9. Optical injection locked phase lock loop [38].

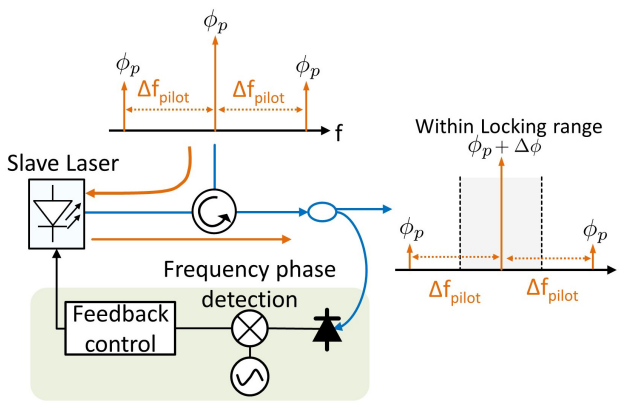

Figure 10. Pilot tone assisted OIL phase locked loop.

\section{B. OIL phase locked loop}

The direct detection feedback control described in the previous section is based on amplitude measurement and as such its accuracy is compromised with drifts, e.g., in the free-running slave laser output power. In applications where broadband phase synchronization is required, it is better to implement an optical phase lock loop (OPLL), forming an OIL-OPLL (abbreviated as OIPLL) [38].

Fig. 9 shows an example of an OIPLL, specifically a heterodyne OIPLL [38]. A portion of the master laser light is modulated by a sinusoidal signal (at an 'intermediate frequency', IF) to create a side tone before injection into the slave laser. The slave laser is aligned and locked to a side-tone to achieve OIL. The unmodulated master light is then combined with the output of the slave laser and is detected by a photodetector, creating a beat signal at the IF. The beat signal is subsequently mixed with the IF oscillator's output for phase error detection. The error signal then passes through a loop filter and feedback to the slave laser bias current for frequency and phase control. In this OIPLL system, the OIL is used to suppress the wide bandwidth phase noise generally reducing the slave laser linewidth significantly. The OPLL then deals with the low frequency phase variation, ensuring a low phase error and long-term 
locking stability. The noise performance of an OIPLL system was analyzed in detail in [38], [60].

\section{Pilot tone assisted OIL phase locking}

Though OIPLL offers superior phase noise performance, the PLL feedback control requires high speed electronics and short loop length to ensure a sufficiently high locking bandwidth, adding cost and complexity. Thus, we proposed a pilot tone assisted scheme that requires only slow electronics to stabilize the OIL even for ultra-low injection ratios of $<50 \mathrm{~dB}$ [3], [50], [61]. The schematic diagram of the proposed method is shown in Fig. 10.

Using reflection type OIL as an example, we add a pilot tone to the master signal. This can be achieved by modulating the master $\mathrm{CW}$ signal or by filtering of a frequency comb [50], [61], [62]. The pilot tone frequency is chosen to be higher than the locking range. Therefore, the output of the slave laser consists of the injection locked slave output and the pilot tone sidebands purely reflected by the slave laser front facet (as they are outside the OIL range). The slave laser output experiences phase shift $\phi$ depending on the $\Delta \omega$ detuning (Fig 4, (7)), while the phase of the reflected pilot tones is not affected by the OIL, as shown in Fig.10. Consequently, the information of $\phi$ (and thus $\Delta \omega$ ) can be extracted by measuring the phase of the beat between the injectionlocked carrier and the pilot tone side-bands, all of them being conveniently present at the slave laser output. After being detected by a photodetector, the radio frequency (RF) beat signal between the carrier and the pilot tone is given by:

$$
I=1+\frac{\eta^{2}}{2}+\frac{\eta^{2}}{2} \cos \left(2 \omega_{p} t\right)+2 \eta \cos \left(\omega_{p} t\right) \cos (\phi)
$$

where $\eta$ is is the normalized intensity ratio between carrier and pilot tone, and $\omega_{p}$ is the angular frequency of the pilot tone. The fourth term of (10) carries the desired information on $\phi$, which is used as an error signal for the PLL after being down-converted to the baseband using an RF mixer.

\section{OIL Directly Modulated Lasers}

Directly modulated lasers have always been used in optical telecommunications [63], but their modulation chirp prevented them from being used for long distances or at very high bit rates. Modern DML combined with advanced modulation formats are capable of data rates in excess of $>100 \mathrm{~Gb} / \mathrm{s}$, albeit over limited distances. Despite these limitations, DMLs are very attractive in cost and size sensitive markets such as data center interconnection or supercomputer or in access networks [64]-[66], as they enable 'minimum optical hardware' (e.g. DML at transmitter side and a single photodiode at the receiver side) implementations. With the increasing need for high baudrate data communications, further improvement in DML bandwidth and link reach is necessary in these fast-developing markets.

Self-feedback and OIL were both studied to enhance the modulation bandwidth and reduce modulation chirp [67]. Up to $50 \mathrm{GHz}$ bandwidth DML has been demonstrated using self-feedback [68], [69]. Compared to the self-feedback (essentially a laser which self-injectionlocks), OIL provides additional degree of freedom in the rate equations, which is frequency detuning $\Delta \omega$. In the following sections, we review the application of highspeed OIL DML for optical communications systems.

\section{A. High-bandwidth DML}

The OIL-DML came into the spotlight in the 1980s of the fast- developing optical communications that was moving to higher bandwidth signals. After setting the theoretical ground work, the features of bandwidth enhancement and chirp suppression were exploited in direct-modulation direct-detection (DM-DD) transmissions. The suppressed chirp and more linear power modulation has led to a two fold increase in transmission distance in a $2 \mathrm{~Gb} / \mathrm{s}$ DM-DD system, which was considered as a significant improvement at that time [33].

Entering the $21^{s t}$ century, the bandwidth enhancement feature of OIL DML was extensively studied with demonstration of up to $40 \mathrm{GHz}$ bandwidth by injection locking a 3-GHz DFB [39]. It has been shown that a more flat frequency response with further bandwidth enhancement up to $60 \mathrm{GHz}$ can be obtained by OILing a slave laser with multiple master lasers [70].

In the past decade, OIL DML in conjunction with digital signal processing (DSP) has produced data transmission beyond $100 \mathrm{~Gb} / \mathrm{s}$ using high order modulation format, at conventional telecomm C-band and also in a new transmission windows [71], [72]. Researchers have also innovated new transmitter architecture using OIL DML to reduce the carrier to signal power ratio to facilitate more power efficient transmission with improved performance [73]. Different types of lasers, such as VCSEL [74], [75] and microring lasers, have also been evaluated with OIL, showing significantly improved data transmission performance [76].

Using VCSEL as both master and slave lasers, Lu demonstrated $45 \mathrm{~Gb} / \mathrm{s}$ PAM4 transmission [74]. Although the experiment was based on free-space coupling, a potential of integrating two VCSELs vertically 
may help to overcome the bandwidth bottleneck of the VCSEL [74], [75]. A more recent research reports OIL to microring lasers [76], obtaining high side mode suppression ratio (SMSR) of up to $56 \mathrm{~dB}$ with intrinsic modulation bandwidth increased from $3 \mathrm{GHz}$ to 18 $\mathrm{GHz}$ for different longitudinal modes across a $30 \mathrm{~nm}$ wavelength range. The microring structure allows for easy OIL in a transmission configuration, eliminating the need for circulators or isolators for potential low-cost and integrated solutions [76].

\section{B. Coherent transmitter based on DML}

Modern transmitters require encoding data on both amplitude and phase of the optical carrier, known as quadrature amplitude modulation (QAM). This is usually achieved by using chirp-less external modulation such as lithium niobate Mach-Zehnder modulators (MZMs) in a nested structure, to modulate both the In-phase (I) and Quadrature $(\mathrm{Q})$ component of optical field. For a long time, it was believed that DML cannot be used to generate QAM signal because of the modulation chirp. The advantages of using DML over the lithium-niobate MZM would be lower insertion loss, reduced power consumption, small footprint and ease of integration. OIL with its ability of modulation chirp reduction and phase synchronization of multiple lasers (one master and one or more slave lasers) opens new opportunities for DML-based QAM signalling.

Fig.11 sketches our method of QAM signal synthesis based on OIL DMLs. For simplicity, we explain the principle of operation using 16 QAM as example. As we will show later, arbitrary amplitude and phase modulated signal can be generated in this manner. The transmitter consists of two slave DMLs, Laser I that is modulated with the I signal component and Laser Q that is modulated with the Q signal component. Both lasers are injection-locked by the same master laser, such that the two DMLs become mutually coherent (OIL to the same master laser) and the modulation chirp is suppressed. The mutual coherence allows us to combine the outputs of the two OIL DMLs coherently. By setting the relative phase between them to $90^{\circ}$, we obtained IQ modulated signal, as indicated by the constellation in Fig.11. Since direct laser modulation is essentially intensity modulation, the constellation of the synthesized signal lies in the first quadrant of the complex plane and its optical spectrum contains a carrier component (the blue arrows in Fig.11). This carrier component can be removed by destructively interfering the combined signal with an unmodulated component of the injection $\mathrm{CW}$ that is obtained by tapping a portion of the master laser a
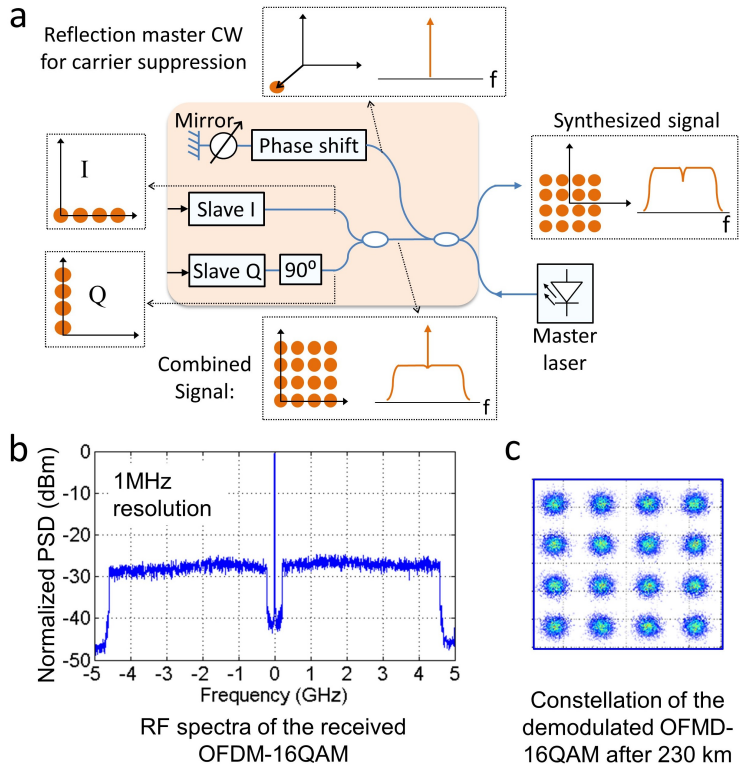

C

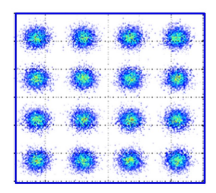

Constellation of the demodulated OFMD 16QAM after $230 \mathrm{~km}$

Figure 11. (a) Schematic diagram of the OIL-DML based coherent transmitter; (b) Spectrum of the generated signal, calculated from the received signal from a coherent receiver; (c) constellation diagram of demodulated OFDM signal after $230 \mathrm{~km}$ transmission.

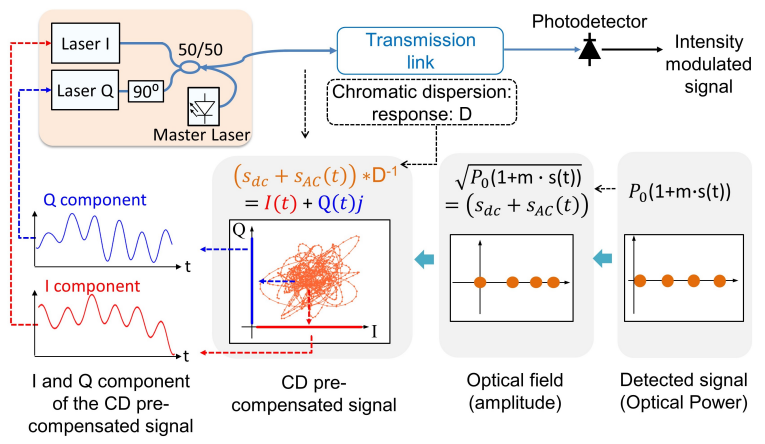

Figure 12. Operation principle of the OIL-based transmitter that allows for amplitude modulation with arbitrary phase profile.

power. When the carrier of the combined signal is fully suppressed, a carrier less QAM signal is obtained.

To show that the transmitter can generate arbitrary amplitude-phase modulated signals, we modulated the two slave lasers with I and Q component of an orthogonal frequency-division multiplexing (OFDM) signal using 16 QAM for each subcarrier. Fig. 11b and Fig. 11c show the spectrum of the detected signal via coherent receiver and the constellation diagrams of the demodulated OFDM-16QAM. Compared to standard $\mathrm{LiNbO}_{3}$ IQ modulator, the transmitter showed similar performance after $230 \mathrm{~km}$ transmission [77], evidencing high signal quality. 
The carrier suppression branch can be removed to form a simplified version of the transmitter, as shown in Fig. 12. Due to the freedom of modulating independently the I and Q components, the simplified scheme can generate amplitude modulated signal with arbitrary phase profile. Thus, it is possible to generate dispersion precompensated amplitude-modulated signals. Using fourlevel pulse amplitude modulation (PAM4) as an example, the ideal received signal has four power levels described by $P_{0}(1+m \cdot s(t))$, where $P_{0}$ is the average power, $\mathrm{m}$ is the modulation index, $s(t)$ is the modulation signal (PAM4 in this case). On the constellation diagram, it has four points located on the real-axis. The corresponding optical field of the PAM signal is therefore $\sqrt{P_{0}(1+m \cdot s(t))}$, it can be decomposed as a DC component $s_{d c}$ plus an AC component $s_{A C}(t)$. Let us assume the optical link is impaired with the chromatic dispersion (CD) only, and that the CD operator (time domain response of total $\mathrm{CD}$ ) is $D$. The optical signal at the transmitter output then should be $\left(s_{d c}+s_{A C}(t)\right) * D^{-1}$, where $D^{-1}$ is the time-domain response of the CD compensation fiber. The precompensated signal lies in the first quadrant and after its decomposition into the Inphase and Quadrature components, it can be modulated onto the two slave lasers (Slave I and Slave Q) to generate the required CD-precompensated signal.

Using this transmitter, we generated CDprecompensated signals and achieved 300-km transmission of PAM4 signal without using any dispersion compensation fiber [78]. In principle, the used transmitter-side DSP allows for compensation of all linear transmission impairments (on the top of the demonstrated $\mathrm{CD})$ ). It is worth reiterating that this unique ability to compensate for transmission impairments is combined with other OIL benefits such as enhanced modulation bandwidth, suppressed modulation chirp, reduced relative intensity noise, and narrow laser linewidth.

The lasers used in these proof-of-concept experiments were low cost Fabry-Perot lasers emitting at many longitudinal modes (having multiple spectral tones) when free-running. Their modulation bandwidth was only 2 $\mathrm{GHz}$. By selection of the master laser wavelngth, it was possible to injection lock different longitudinal modes, making the slave lasers lasing at one longitudinal mode only (making them effectively single line) with having that 'single-line' emission tuneable over the entire Cband. Similar structure was demonstrated using VCSELs, showing phase shift keyed (PSK) modulation at 10 $\mathrm{Gb} / \mathrm{s}$ for quadruple PSK (QPSK) and at $40 \mathrm{~Gb} / \mathrm{s}$ binary PSK (BPSK) [79], [80].

Coherent transmitters have made significant progress

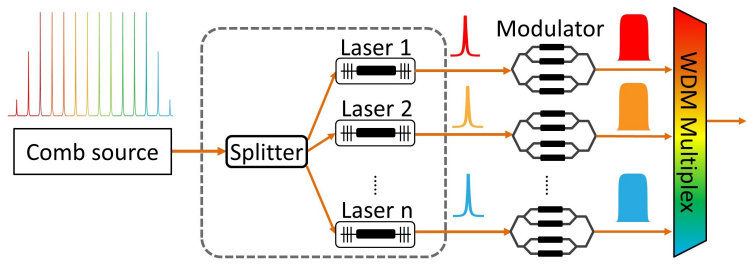

Figure 13. Schematic of the OIL based super-channel transmitter.

in the last few years. Integrated photonic devices with $40 \mathrm{GHz}$ bandwidth and beyond have been demonstrated using InP, Silicon, and more recently thin-film $\mathrm{LiNbO}_{3}$ [81]. There is a long way for DML based coherent transmitters to catch up for applications in telecommunications. Yet it is worth discussing the pros and cons of this fundamentally different technique compared to other full-field modulation methods.

\section{OPTICAL CARRIER RECOVERY}

The features of linewidth narrowing and low-noise single tone amplification make OIL an attractive solution for the selection and recovery of optical carriers for coherent optical communications. At the transmitter side, OIL has been identified as an effective method to select and amplify individual comb tones, overcoming the issue of low power per tone for frequency comb based superchannel signal transmission [82]-[86]. At the receiver side, OIL was exploited to recover optical carriers in the optical domain to enable low complexity and low latency homodyne coherent communications [62], [87][89]. In a transmission link, OIL was demonstrated in phase sensitive amplification, which in principle can achieve zero noise figure or signal regeneration. Besides communications, carrier recovery function of OIL is used to isolate and manipulate individual comb tones for arbitrary waveform generation, sensing, and terahertz signal generation [53], [90]. In the following section we discuss these applications.

\section{A. Coherent super-channel transmitter}

Optical frequency combs offer coherent tones with a fixed frequency spacing. Originally employed in metrology, it has also been shown that DWDM transmission systems with mutually frequency-locked optical carriers can potentially offer several advantages as compared to conventional laser-bank based DWDM systems, including higher spectral efficiency, bandwidth flexibility, and efficient compensation of fiber nonlinearity [91]. These performance aspects are highly relevant for future high capacity, flexible grid optical networks. An integrated 
transmitter with frequency-locked optical carriers could provide an attractive approach in this context.

In DWDM transmission, comb tones must be extracted and individually modulated. An ideal comb source should be compact, wide band, and supply uniform $>10$ $\mathrm{dBm}$ power per tone. However, in practice, compact comb sources often generate tones with small and unequal powers [57], [92]-[94]. To boost the power of each tone and equalize their powers, a programmable optical filter (e.g. a Waveshaper) followed by an optical amplifier is typically used [92]-[94]. This inevitably degrades the OSNR and makes the transmitter integration difficult. In light of these issues, researchers are starting to exploit OIL to select and amplify the comb tones for superchannel transmitters.

The general structure of the investigated transmitter is shown in Fig. 13. The frequency comb is injected into the slave lasers. The extracted tones are modulated individually via IQ modulators for signal generation. Due to the fixed tone spacing, the guard band can be minimized [7]. The phase coherent sub-channels can also be synthesized to become a large bandwidth super channel provided the phase between different paths are locked [95].

In [6], Albores-Mejia compared the system performance of the OIL method with the filter + amplifier configuration for tone selection and amplification. They showed that even when the comb is significantly attenuated to about $-35 \mathrm{dBm}$ per tone, OIL could still regenerate the selected comb tones with sufficiently high performance to support 12 Gbaud dual-polarization 64QAM transmission, outperforming the filter + amplifier method. The tone recovery with low comb power allows the comb to be distributed to a large number (e.g., 100s) of racks for both short and long-reach coherent communications. In [7], we OIL a telecom-grade InPbased digital supermode distributed Bragg reflector (DS$\mathrm{DBR}$ ) tunable laser to a $25-\mathrm{GHz}$-spaced frequency comb, showing C-band tunable tone extraction with a simultaneous linewidth reduction from $1.5 \mathrm{MHz}$ to $4 \mathrm{kHz}$. Importantly, our experiment showed that the key building blocks (inc. comb [96], tunable laser, modulator) are readily achievable on the InP photonic integration platform. Low injection ratio also helped reducing the reflected optical signals to keep high SMSR without any optical pre-filtering [9].

Temprana [8] demonstrated the benefit of OIL-based transmitters in long-reach coherent systems. Frequency referenced optical carriers were generated by injection locking DFB lasers to a reference optical frequency comb. The frequency referenced tones harnessed the potential of transmitter-side digital back propagation

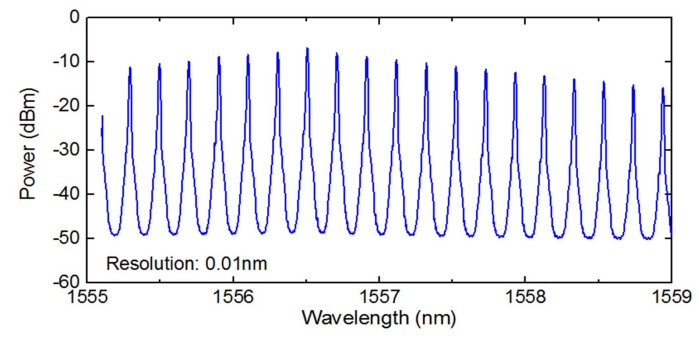

Comb injection from rear facet

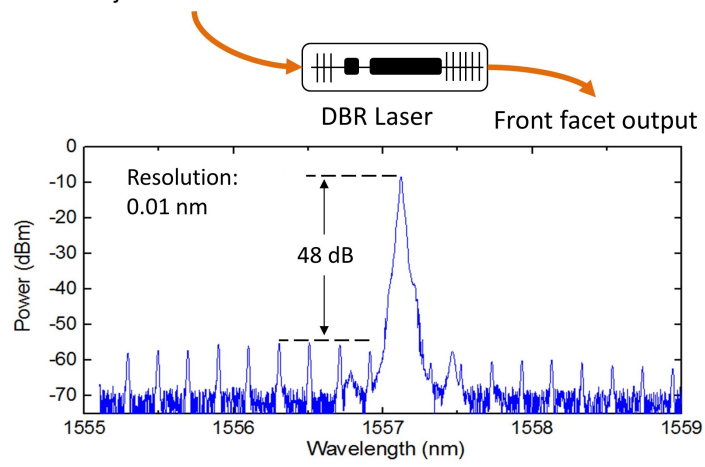

Figure 14. Rear facet OIL that allows for high SMSR.

(DBP), enabling two-fold increase in system reach, from 1530 to $3060 \mathrm{~km}$, in a four channel 16 GBaud 16 QAM system [8].

Despite the successful demonstrations of the concepts, there are still several problems to be addressed. Firstly, isolators/circulators are not available in photonic integrated circuit (PIC) platforms. Thus, research on PIC isolators or circulators would largely benefit the system application. Another potential solution is to engineer the slave laser such that the light from the slave laser does not feedback to the master comb laser. Secondly, the limited SMSR causes crosstalk to other channels. A promising method is to injection lock the slave laser from the rear facet. As we show in Fig.14, a high finesse transmission response of a laser cavity (e.g. FP cavity) can largely reduce the side modes for regeneration of a single mode with high SMSR. Our preliminary research has shown that up to $48 \mathrm{~dB}$ SMSR is possible with rearfact OIL.

\section{B. Optical local oscillator recovery}

Coherent detection requires synchronizing frequency and phase of the local oscillator to the received signal for amplitude and phase recovery. This frequency offset (CFO) recovery and carrier phase recovery (CPR) functions are usually realized in DSP after coherent detection and digitization (analog-to-digital conversion, ADC). Indeed, DSP offers unparalleled possibilities in 


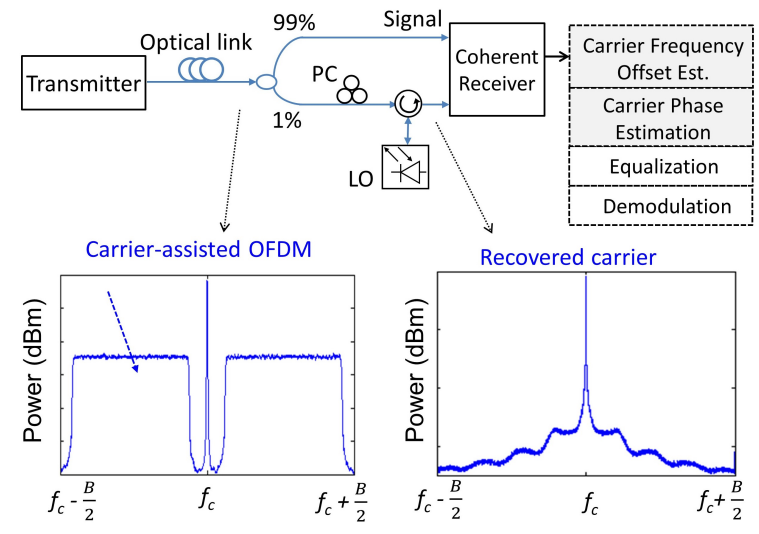

Figure 15. Concept of OIL based optical carrier recovery in OFDM transmission.

the manipulation of signals and have led to the resurrect of coherent communications in the last 15 years [97].

However, the use of DSP may be undesirable in midspan regeneration, in which the signal is further transmitted on an optical carrier and it is often simply not possible to use it, e.g., in quantum optics. The use of DSP and ADC also increase power consumption and latency, which is highly-undesirable in many applications such as high-frequency trading, 5G mobile communications [98], and low latency coherent data center interconnection [99], [100]. In these areas, phase locking of the local oscillator to the incoming signal in the optical domain could be beneficial to coherent detection. As discussed in Section IV, OIL can effectively phase lock a slave laser (e.g. LO) to a master light (e.g. received signal) for homodyne coherent detection, as shown in Fig.15. This potentially eliminates the need for carrier frequency offset (CFO) and carrier phase estimation (CPE) in DSP. Furthermore, OIL also turn a large linewidth low cost LO into a narrow linewidth LO (linewidth narrowing feature discussed in section III-a), enabling high order modulation formats with the reduced total linewidth.

However, the absence of the signal carrier in coherent modulation formats (e.g. QPSK, 16QAM and higher) poses a major challenge for optical carrier recovery techniques (e.g. OPLL, OIPLL) for phase synchronisation between the LO and the data signal [101]. To resolve this problem, line coding such as $8 \mathrm{~B} 10 \mathrm{~B}$ was used to create a gap in the center of the signal spectrum, where a $\mathrm{CW}$ tone can be inserted to co-propagate with the modulated side bands [102].

The OFDM modulation format, in which a carrier tone is usually inserted in the middle of the OFDM spectrum (carrier-aided OFDM), does not require additional line coding. The optical carrier tone can be generated by biasing the optical modulator slightly away from the transmission null and it has been shown that residual carrier tone benefits coherent receiver by simplifying the DSP-based CFO, CPE, and fiber nonlinearity compensation [103], [104]. Moreover, a guardband that isolates the carrier tone from the data can be easily created by setting the low frequency OFDM sub-carriers to zero. All these features make OFDM a promising format for OILbased homodyne detection (as first suggested in [105]). The early proof-of-principle demonstration required a relatively large protection guardband (reducing transmission capacity by pre-filtering before the OIL). This prefiltering was later shown to be unnecessary provided the locking range is sufficiently small. Injection ratio as low as $-65 \mathrm{~dB}$ [3] was the key enabler of this system. In addition to optical fiber communications, OIL based homodyne coherent detection is also exploited in free space communications. Kakarla [51] demonstrated sub$\mathrm{nW}$ injection powers, although with the help of an EDFA to pre-amplify the low power signal.

The carrier tone can also be placed at the side band, as shown in [57]. This eliminates the need for creating a guardband for single-carrier modulation format. After the carrier recovery, the recovered LO needs to be converted back to the center for homodyne coherent detection by single sideband frequency shifting techniques.

OIL can also be used to recover LOs in a comb based transceiver. In [88], [106], one (or two) of the comb tones were left unmodulated, while the rest of the tones were modulated individually to generate QAM signals. The unmodulated tones served as pilots that were recovered via OIL at the receiver side. The recovered $\mathrm{CW}$ signal then seeded an electro-optic comb generator [107]. In these type of transceivers, the phase-locked nature of the optical carriers are exploited in the DSP for the DWDM signals, showing reduced DSP complexity [5]. OIL is crucial in these systems as it regenerates CW tones with high OSNR. The reduced linewidth ensures a low phase noise clock recovery from the beating of the tones, ensuring high quality signal detection.

Yet there is still a major challenge to use OIL at the receiver side. How to make it polarization insensitive. The easiest way is to track the polarization [108]. Alternatively, a polarization insensitive method was proposed in [89], [109].

\section{Phase sensitive Amplifiers and Parametric comb gen- eration}

OIL has been very popular in Phase Sensitive Amplification (PSA). A PSA amplifier has gain dependent on the phase of the signal, as opposed to traditional optical 


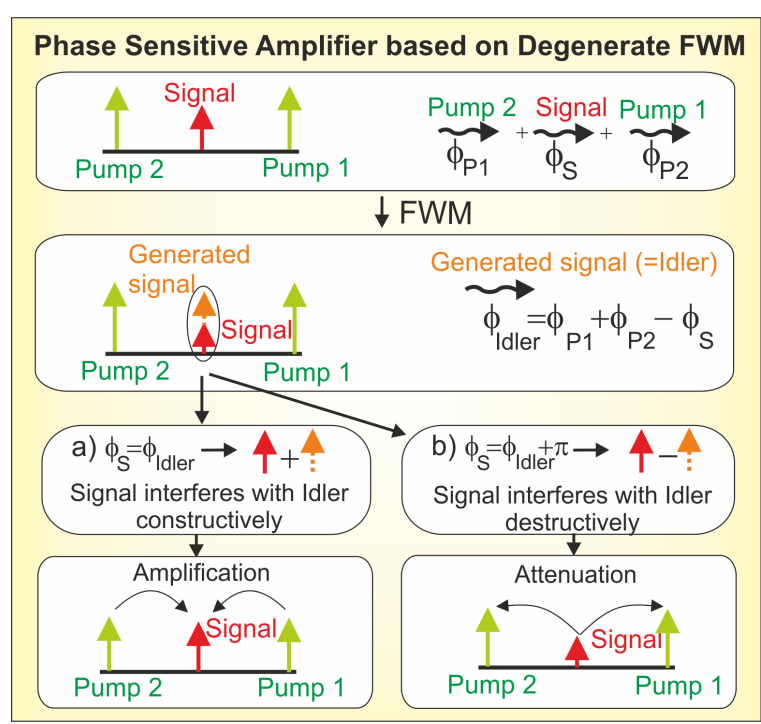

Figure 16. PSA implementation through a degenerate FWM process. The PSA maximally amplifies the signal when its phase is 0 or $\pi$ relative to the phase of the pumps, and maximally attenuates the signals when the phases are $\pi / 2$ or $3 \pi / 2$.

amplifiers (e.g. EDFA), which amplify an input signal irrespective of its phase. An example of PSA based on degenerated FWM is shown in Fig. 16. This particular PSA was demonstrated to be used as a regenerator of BPSK signals [110], [111], since it amplifies signals with phases of 0 or $\pi$ (where data is carried), while it attenuates signal components that have orthogonal phases (where there is only noise).

Apart from regeneration of binary PSK signals, PSAs were also demonstrated to regenerate more complicated modulation formats such as QPSK [112], quantize optical phase [113], performing almost noise-less data amplification [114], [115], noise-suppressed amplification of optical frequency combs [116], etc.

The main challenge of most PSA-based signal processors is to generate a low-noise, high-power pump that would be phase-locked to the (noisy) data. OIL is an ideal technique to perform this task. Let us demonstrate it on the example of the first PSA-based optical regenerator that needed only the noisy data signal at its input (requiring the pumps for the FWM process to be generated within the regenerator) [111]. The schematics of generating pumps is shown in Fig. 17. Firstly, a free running laser (Pump 1) is mixed with the noisy data signal in a non-linear medium (highly nonlinear fiber (HNLF) here), generating an Idler that is phase-locked to the Signal and Pump 1 (due to the nature of the FWM). Although the Idler is modulation free due to the effect of modulation stripping [111], it contains all the phase and

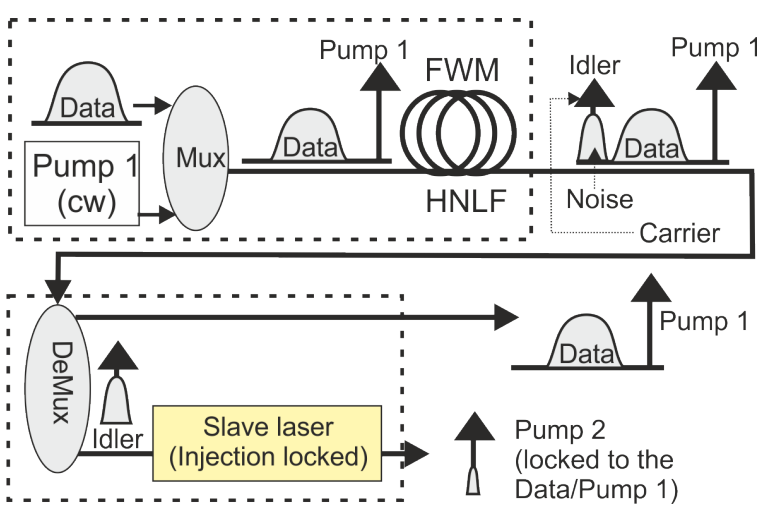

Figure 17. Phase synchronization of the pumps to the data signal in a PSA-based optical regenerator. HLNF: Highly non-linear fiber.

amplitude noise present on the data Signal, making it unsuitable as the Pump 2 in the PSA. Therefore, OIL is used with Idler as the master - removing all the amplitude noise and strongly suppressing all the phase noise that is out of the locking range. Typically, data Signal has bandwidth of 10 s of $\mathrm{GHz}$ (e.g., $\pm 40 \mathrm{GHz}$ in [111], while OIL is set to have sub-GHz bandwidth, filtering out majority of the noise.

In some PSA implementations [114], [115], the pump signal is transmitted along with the data Signal, but needs to be regenerated prior to PSA. OIL is often used in this scenario too for the same benefits as outlined above.

Apart from FWM-based PSAs, OIL has been used in other parametric (e.g., FWM) based processes, e.g., to generate optical frequency combs of unique properties (e.g., with very high optical signal-to-noise ratio). This was first demonstrated in [117], Fig. 18. Side-modes are generated from a CW laser via an electro-optic modulator first. Subsequently, two of them are OIL to generate a strong and low-noise two-tone signal with the two tones phase locked together. In the next step, these two tones are sent through a nonlinear medium (such as HNLF), generating new spectral components (via FWM). The generated frequency comb can be investigated in the temporal domain, producing, e.g., short optical pulses (such as in [117]) or in the frequency domain as a bank of phase-locked optical frequency carriers [118]. In the latter case, over $100 \mathrm{~nm}$ wide frequency combs were achieved via precise engineering of new tones generation in the engineered HNLF. For example, in an early work [118], 100-GHz spacing covering $100 \mathrm{~nm}$ bandwidth $(>120$ tones) with $>5 \mathrm{dBm}$ per tone and optical signal-to-noise ratio OSNR $>42 \mathrm{~dB}(0.1 \mathrm{~nm}$ bandwidth) was shown in a variety of applications (e.g., [91], [119], [120]) and has been also commercialised by RAM Photonics, LLC. 


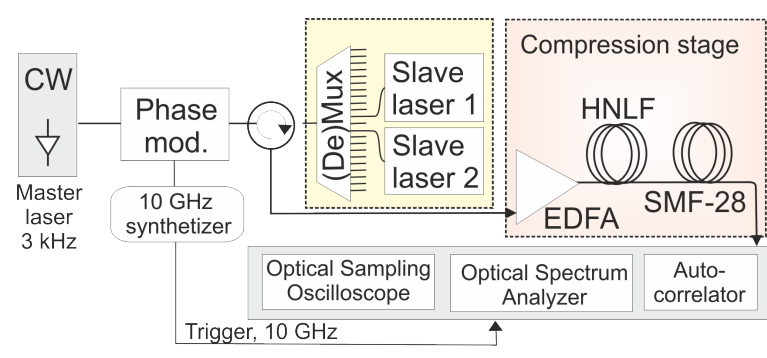

Figure 18. Generation of optical frequency comb, which produced short optical pulses in the temporal domain.

\section{Optical waveform synthesis}

In optics, generation of customized-shaped pulses generally consist of shaping pulses generated, e.g., by a mode-locked laser. The most flexible technique consists of performing shaping of the amplitude and phase of the pulse spectrum using spatial light modulator and a $4 \mathrm{f}$ arrangement (so called Fourier synthesis, as the $4 \mathrm{f}$ interferometer performs optical Fourier transform, processes pulse spectrum, and a subsequent inverse optical Fourier transform back to temporal domain) [121]. In its ultimate implementation, it performs 'line-by-line' spectral shaping [122]. As the repetitive pulse source (such as a mode-locked laser) have spectrum consisting of discrete tones (with spacing corresponding to the repetition rate), manipulating each tone individually (rather than group of tones performed in earlier Fourier synthesis) gives ultimate flexibility enabling arbitrary amplitude/phase pulse shaping. These pulse shaping techniques, however, are based on attenuation/elimination of spectral components/tones and manipulation of their phases. As discussed in section III-B, OIL can be used to selectively amplify spectral components of interest (rather than attenuate unwanted spectral components), even from a pulsed source of relatively small repetition rate, such as metrology-grade optical frequency comb. In [50], this was shown using a pulsed source with repetition rate (tone spacing) as small as $250 \mathrm{MHz}$, requiring very little power of the tone-to-be-locked to (down to $-60 \mathrm{dBm}$ ), enabling direct comb tone amplification (by $65 \mathrm{~dB}$ ) without using any EDFA amplification. Pulse synthesis via OIL of multiple lasers and tunable alignment of their relative amplitudes and phases was shown in [11], Fig. 19.

OIL can be also used for generating RF waveforms, as shown, e.g., in [123], where RF waveform tunable over seven octaves was demonstrated. This technique is based on deep modulation of an optical carrier (which generates higher-order modulation sidebands), subsequent OIL to one of the higher order modulation sidebands, and
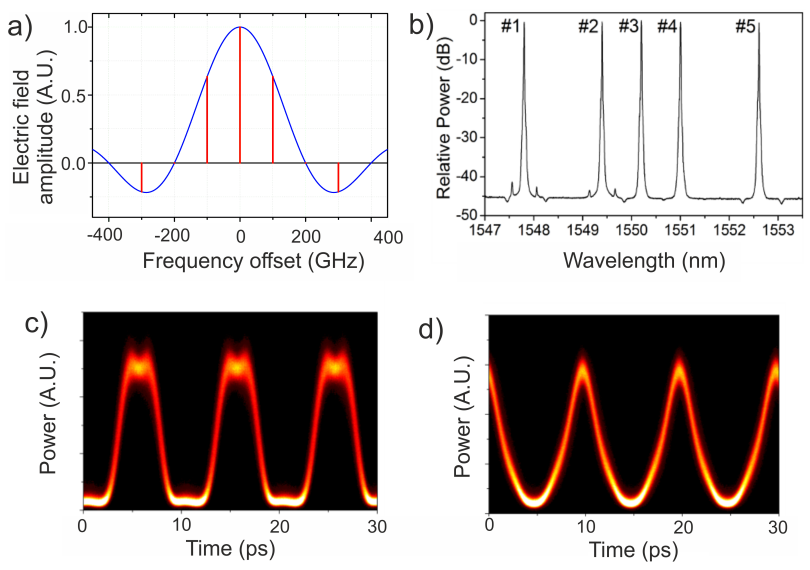

Figure 19. (a) Illustration of a sinc-shaped spectrum envelope (blue) realized with five discrete non-zero components (red). (b) OIL of five lasers used to realize (by subsequent phase/amplitude manipulation) of the sinc-shaped spectrum (shown in in (a)). Experimental result for (c) flat-top and (d) inverse-parabolic pulses generated via manipulation of phases/amplitudes of the five lasers.

finally beating of the original carrier and the OIL laser on a photodetector.

\section{E. Transfer of precise frequency}

The ultra-precise optical frequency standards have been improved progressively down to the frequency instability of a few parts in $10^{-18}$ [124]. For their distribution (or comparison), it is necessary to use more precise means than the traditional satellite-based frequency transfer. Transmission over optical fibers has emerged as such an alternative with distances up to 1840 $\mathrm{km}$ achieved with frequency instability down to $10^{-19}$ level [125]. This requires bi-directional propagation of signals through optical fibers, which enables cancellation of the fiber drift (that otherwise causes Doppler shift of the transmitted frequency). Amplification of such signals (essentially $\mathrm{CW}$ lasers with carrier frequency locked to a frequency standard) over bi-directionally operated fiber is challenging, especially due to the need of amplification (e.g., EDFAs tend to cause parasitic lasing when operated bi-directionally, etc.).

OIL is an ideal 'amplifier' of signal that carries precise frequency (essentially a CW laser). Firstly, it provides very high amplification (e.g., > $60 \mathrm{~dB}$ ). Simultaneously, it can be designed (using very low injected powers, Fig. 4) to amplify signal over very limited locking range, rejecting higher-frequency noise picked up by the signal during propagation, performing regeneration. This regeneration is 'out of band' for both, amplitude and phase. Finally, for low injection powers, slave laser output power is essentially insensitive to the injected master 
power, providing (in-band) amplitude regeneration too. This has been demonstrated in [61], where it enabled frequency transfer over $292 \mathrm{~km}$ of installed fiber with fractional frequency instability below $10^{-19}$.

OIL could be also used for wavelength conversion of a CW laser that carries information about precise frequency [126]. This is useful for frequency transfer over telecom optical network in which the same wavelength channel may not be available over the entire network and thus wavelength conversion must be adopted. In [126], the signal was first regenerated with the OIL, which seeded and electro-optic frequency comb with $25 \mathrm{GHz}$ comb spacing. Second slave laser was OIL to the comb tone of interest (e.g., 8th comb tone from the carrier for wavelength conversion over $200 \mathrm{GHz}$ ), effectively performing wavelength conversion.

\section{FUtURE RESEARCH DIRECTIONS}

In the last decade, OIL technology has matured and is in our view ready for commercial applications, e.g., in various systems discussed in sections $\mathrm{V}$ and VI. Although research on OIL has been going on for more than half a century, new scientific insight into OIL phenomenon and innovate new applications keep appearing, opening new exciting opportunities for OIL and OIL based systems.

On the laser dynamics front, novel laser material research (e.g. quantum dots) requires new scientific understanding on the OIL laser dynamics. For example, the low/negative linewidth enhancement factor available with the quantum dot material could significantly reduce the modulation chirp and provide symmetric locking region [45]. New laser designs involving silicon photonics have demonstrated new features such as an extended photon lifetime for significant reduction of the intrinsic laser linewidth [127]. OIL features of these new lasers are yet to be studied.

On the OIL applications front, we advocate that system researchers should work together with laser designers rather than in isolation, which is the norm today. A commercial laser without the isolator used for OIL is very likely to have poorer performance than a laser specifically designed for the intended OIL application. An example is the large-bandwidth OIL lasers, in which we need to obtain simultaneously high resonance frequency and flat frequency response, which requires simultaneous optimization of bandwidth, damping, frequency detuning, and OIL ratio.

In addition to laser engineering, applications impose challenges on integration and polarization sensitivity. In optical transceivers, it is preferred to have both master and slave lasers on the same chip for OIL
DMLs and carrier recovery. However, this has always been a challenge due to the lack of optical isolators on the PIC platforms. On-chip isolators would largely benefit the OIL based systems. Another solution may be the newly emerged reflection-insensitive lasers [128]. Yet another approach would be to design master-slave pair considering the back-reflection into master in the design (by adding relevant term into the rate equations). This would be similar to the example of two coupled pendulums affecting each other.

Even without the back-reflection solution, there is a large class of applications in which a single master (CW laser or an optical frequency comb) is used, requiring only single isolator between the master and (potentially multiple) slave lasers, e.g., for Fourier waveform synthesis. In applications requiring optical carrier recovery, (e.g. the LO recovery we discussed in Section VI-B), the OIL process needs to cope with input signal polarization variations, making research on polarisation insensitive OIL crucial for these applications [89].

\section{CONCLUSION}

OIL has been demonstrated and studied for more than fifty years. Standing on the shoulders of giants, engineers and researchers can now harness the useful features it offers, enabling new applications in optical communications and signal processing. To get the full benefit of OIL, intuitive understanding and stable operation are crucial for practical applications and innovations. In this paper, we attempt to explain OIL features without diving deep into the OIL rate equations. Together with the control methods summarized in Section IV, we hope to introduce the OIL technique to a wide range of researchers and engineers, especially those who are not familiar with it. OIL has entered numerous applications in the past decade, yet the OIL based applications are far more than those we introduced. Exciting new opportunities and challenges lie ahead waiting for passion and new ideas.

\section{APPENDIX}

$\begin{array}{ll}\text { BPSK } & \text { Binary phase shift keying } \\ \text { CD } & \text { Chromatic dispersion } \\ \text { CFO } & \text { Carrier freuqency offset } \\ \text { CPE } & \text { Carrier phase estimation } \\ \text { CW } & \text { Continuous wave } \\ \text { DBR } & \text { Distributed Bragg reflector }\end{array}$




$\begin{array}{ll}\text { DCF } & \text { Dispersion compensation fiber } \\ \text { DFB } & \text { Distributed feedback } \\ \text { DML } & \text { Directly modulated laser } \\ \text { DM-DD } & \text { Direct modulation direct detection } \\ \text { DSP } & \text { Digital signal processing } \\ \text { DWDM } & \text { Dense wavelength division multiplexed } \\ \text { EDFA } & \text { Erbium-doped fiber amplifier } \\ \text { FP } & \text { Fabry Perot } \\ \text { FWM } & \text { Four wave mixing } \\ \text { HNLF } & \text { Highly nonlienar fiber } \\ \text { LO } & \text { Local oscillators } \\ \text { RF } & \text { Radio frequency } \\ \text { IM-DD } & \text { Intensity modulation direct detection } \\ \text { OFDM } & \text { Orthogonal frequency division multiplexin } \\ \text { OIL } & \text { Optical injection locking } \\ \text { OPLL } & \text { Optical phase lock loop } \\ \text { OSNR } & \text { Optical signal-to-noise ratio } \\ \text { PAM } & \text { Pulse amplitude modulation } \\ \text { PSA } & \text { Phase sensitive amplification } \\ \text { PSK } & \text { Phase shift keying } \\ \text { QAM } & \text { Quadrature amplitude modulation } \\ \text { QPSK } & \text { Quadrature phase shift keying } \\ \text { SOA } & \text { Semiconductor optical amplifier } \\ \text { SMF-28 } & \text { Standard single mode fiber } \\ \text { SMSR } & \text { Side mode suppression ratio } \\ \text { VCSEL } & \text { Vertical cavity surface emitting laser } \\ \text { WSS } & \text { Wavelength select switch } \\ & \quad \text { REFERENCES } \\ & \end{array}$

[1] A. E. Siegman, Lasers. Mill Valley, CA: University Science Books, 1986, ch. 29.

[2] K. Kikuchi, T. Okoshi, M. Nagamatsu, and N. Henmi, "Degradation of bit-error rate in coherent optical communications due to spectral spread of the transmitter and the local oscillator," J. Lightw. Technol., vol. 2, no. 6, pp. 1024-1033, 1984.

[3] Z. Liu, J.-Y. Kim, D. S. Wu, D. J. Richardson, and R. Slavik, "Homodyne ofdm with optical injection locking for carrier recovery," J. Lightw. Technol., vol. 33, no. 1, pp. 34-41, 2014.

[4] K. Kasai, Y. Wang, S. Beppu, M. Yoshida, and M. Nakazawa, "80 gbit/s, $256 \mathrm{qam}$ coherent transmission over $150 \mathrm{~km}$ with an injection-locked homodyne receiver," Opt. Express, vol. 23, no. 22, pp. 29 174-29 183, 2015.

[5] L. Lundberg, M. Karlsson, A. Lorences-Riesgo, M. Mazur, J. Schröder, and P. A. Andrekson, "Frequency comb-based wdm transmission systems enabling joint signal processing," Applied Sciences, vol. 8, no. 5, p. 718, 2018.

[6] A. Albores-Mejia, T. Kaneko, E. Banno, K. Uesaka, H. Shoji, and $\mathrm{H}$. Kuwatsuka, "Optical-comb-line selection from a lowpower/low-osnr comb using a low-coherence semiconductor laser for flexible ultra-dense short range transceivers," in Optical Fiber Communication Conference. Optical Society of America, 2015, pp. W2A-23.

[7] Z. Liu, S. Farwell, M. Wale, D. J. Richardson, and R. Slavík, "Inp-based optical comb-locked tunable transmitter," in Optical Fiber Communication Conference. Optical Society of America, 2016, pp. Tu2K-2.

[8] E. Temprana, E. Myslivets, B. P.-P. Kuo, N. Alic, and S. Radic, "Transmitter-side digital back propagation with optical injection-locked frequency referenced carriers," J. Lightw. Technol., vol. 34, no. 15, pp. 3544-3549, 2016.
[9] R. Zhou, T. Shao, M. D. G. Pascual, F. Smyth, and L. P. Barry, "Injection locked wavelength de-multiplexer for optical comb-based nyquist wdm system," IEEE Photon. Technol. Lett., vol. 27, no. 24, pp. 2595-2598, 2015.

[10] T. K. Paraïso, I. De Marco, T. Roger, D. G. Marangon, J. F. Dynes, M. Lucamarini, Z. Yuan, and A. J. Shields, "A modulator-free quantum key distribution transmitter chip," NPJ Quantum Information, vol. 5, no. 1, p. 42, 2019.

[11] D. S. Wu, D. J. Richardson, and R. Slavík, "Optical fourier synthesis of high-repetition-rate pulses," Optica, vol. 2, no. 1, pp. 18-26, 2015.

[12] S. P. Bhooplapur, A. Klee, and P. J. Delfyett, "Line-by-line pulse-shaping at ghz modulation frequencies with an injectionlocked vcsel array," IEEE Photon. Technol. Lett., vol. 29, no. 15, pp. 1241-1244, 2017.

[13] R. A. York and T. Itoh, "Injection-and phase-locking techniques for beam control [antenna arrays]," IEEE Trans. Microw. Theory Techn., vol. 46, no. 11, pp. 1920-1929, 1998.

[14] C. Lin and F. Mengel, "Reduction of frequency chirping and dynamic linewidth in high-speed directly modulated semiconductor lasers by injection locking," Electron. Lett, vol. 20, no. 25, pp. 1073-1075, 1984.

[15] S. Mohrdiek, H. Burkhard, and H. Walter, "Chirp reduction of directly modulated semiconductor lasers at $10 \mathrm{gb} / \mathrm{s}$ by strong $\mathrm{cw}$ light injection," J. Lightw. Technol., vol. 12, no. 3, pp. 418-424, 1994.

[16] G. Yabre, "Effect of relatively strong light injection on the chirpto-power ratio and the $3 \mathrm{db}$ bandwidth of directly modulated semiconductor lasers," J. Lightw. Technol., vol. 14, no. 10, pp. 2367-2373, 1996.

[17] K. Iwashita and K. Nakagawa, "Suppression of mode partition noise by laser diode light injection," IEEE Trans. Microw. Theory Techn., vol. 30, no. 10, pp. 1657-1662, 1982.

[18] T. Simpson, J. Liu, and A. Gavrielides, "Bandwidth enhancement and broadband noise reduction in injection-locked semiconductor lasers," IEEE Photon. Technol. Lett., vol. 7, no. 7, pp. 709-711, 1995.

[19] F. Mogensen, H. Olesen, and G. Jacobsen, "Locking conditions and stability properties for a semiconductor laser with external light injection," IEEE J. Quantum Electron., vol. 21, no. 7, pp. 784-793, 1985.

[20] X. J. Meng, T. Chau, and M. C. Wu, "Improved intrinsic dynamic distortions in directly modulated semiconductor lasers by optical injection locking," IEEE Trans. Microw. Theory Techn., vol. 47, no. 7, pp. 1172-1176, 1999.

[21] H. Stover and W. Steier, "Locking of laser oscillators by light injection," Applied Physics Letters, vol. 8, no. 4, pp. 91-93, 1966.

[22] J.-M. Liu and T. Simpson, "Four-wave mixing and optical modulation in a semiconductor laser," IEEE J. Quantum Electron., vol. 30, no. 4, pp. 957-965, 1994.

[23] J. Sacher, D. Baums, P. Panknin, W. Elsässer, and E. O. Göbel, "Intensity instabilities of semiconductor lasers under current modulation, external light injection, and delayed feedback," Physical Review A, vol. 45, no. 3, p. 1893, 1992.

[24] C. Huygens, Euvres complètes de Christiaan Huygens: Tome VIII Correspondance 1676-1684. Martinus Nijhoff, 1899, vol. 1.

[25] J. Vincent, "On some experiments in which two neighbouring maintained oscillatory circuits affect a resonating circuit," Proceedings of the Physical Society of London, vol. 32, no. 1, p. 84, 1919.

[26] R. Adler, "A study of locking phenomena in oscillators," Proceedings of the IRE, vol. 34, no. 6, pp. 351-357, 1946.

[27] L. Paciorek, "Injection locking of oscillators," Proceedings of the IEEE, vol. 53, no. 11, pp. 1723-1727, 1965.

[28] T. H. Mainman, "Stimulated Optical Radiation in Ruby," Nature, vol. 187, no. 4736, pp. 493-494, aug 1960. 
[29] R. Pantell, "The laser oscillator with an external signal," Proceedings of the IEEE, vol. 53, no. 5, pp. 474-477, 1965.

[30] C. J. Buczek, R. J. Freiberg, and M. Skolnick, "Laser injection locking," Proceedings of the IEEE, vol. 61, no. 10, pp. 1411$1431,1973$.

[31] S. Kobayashi and T. Kimura, "Coherence of injection phaselocked algaas semiconductor laser," Electron. Lett, vol. 16, no. 17 , pp. 668-670, 1980.

[32] R. Lang, "Injection locking properties of a semiconductor laser," IEEE J. Quantum Electron., vol. 18, no. 6, pp. 976-983, 1982.

[33] N. Olsson, H. Temkin, R. Logan, L. Johnson, G. Dolan, J. van der Ziel, and J. Campbell, "Chirp-free transmission over $82.5 \mathrm{~km}$ of single mode fibers at $2 \mathrm{gbit} / \mathrm{s}$ with injection locked dfb semiconductor lasers," J. Lightw. Technol., vol. 3, no. 1, pp. 63-67, 1985.

[34] S. Kobayashi and T. Kimura, "Optical phase modulation in an injection locked algaas semiconductor laser," Electron. Lett, vol. 18, no. 5, pp. 210-211, 1982.

[35] O. Lidoyne, P. Gallion, and D. Erasme, "Analysis of a homodyne receiver using an injection-locked semiconductor laser," $\mathbf{J}$. Lightw. Technol., vol. 9, no. 5, pp. 659-665, 1991.

[36] G. Yabre, "Effect of relatively strong light injection on the chirpto-power ratio and the $3 \mathrm{db}$ bandwidth of directly modulated semiconductor lasers," J. Lightw. Technol., vol. 14, no. 10, pp. 2367-2373, 1996.

[37] T. Simpson and J. Liu, "Enhanced modulation bandwidth in injection-locked semiconductor lasers," IEEE Photon. Technol. Lett., vol. 9, no. 10, pp. 1322-1324, 1997.

[38] A. Bordonalli, C. Walton, and A. J. Seeds, "High-performance phase locking of wide linewidth semiconductor lasers by combined use of optical injection locking and optical phase-lock loop," J. Lightw. Technol., vol. 17, no. 2, p. 328, 1999.

[39] E. K. Lau, X. Zhao, H.-K. Sung, D. Parekh, C. Chang-Hasnain, and M. C. Wu, "Strong optical injection-locked semiconductor lasers demonstrating, 100-ghz resonance frequencies and 80ghz intrinsic bandwidths," Opt. Express, vol. 16, no. 9, pp. 6609-6618, 2008.

[40] C.-H. Chang, L. Chrostowski, and C. J. Chang-Hasnain, "Injection locking of vcsels," IEEE J. Sel. Topics Quantum Electron., vol. 9, no. 5, pp. 1386-1393, 2003.

[41] A. Murakami, K. Kawashima, and K. Atsuki, "Cavity resonance shift and bandwidth enhancement in semiconductor lasers with strong light injection," IEEE J. Quantum Electron., vol. 39, no. 10, pp. 1196-1204, 2003.

[42] E. K. Lau, H.-K. Sung, and M. C. Wu, "Scaling of resonance frequency for strong injection-locked lasers," Opt. Lett., vol. 32, no. 23, pp. 3373-3375, 2007.

[43] E. K. Lau, L. J. Wong, and M. C. Wu, "Enhanced modulation characteristics of optical injection-locked lasers: A tutorial," IEEE J. Sel. Topics Quantum Electron., vol. 15, no. 3, pp. 618633, 2009.

[44] C. Sun, D. Liu, B. Xiong, Y. Luo, J. Wang, Z. Hao, Y. Han, L. Wang, and H. Li, "Modulation characteristics enhancement of monolithically integrated laser diodes under mutual injection locking," IEEE J. Sel. Topics Quantum Electron., vol. 21, no. 6, pp. 628-635, 2015.

[45] Z. Zhang, D. Jung, J. Norman, W. W. Chow, and J. E. Bowers, "Linewidth enhancement factor in inas/gaas quantum dot lasers and its implication in isolator-free and narrow linewidth applications," IEEE J. Sel. Topics Quantum Electron., 2019.

[46] W. Liang, V. Ilchenko, A. Savchenkov, A. Matsko, D. Seidel, and L. Maleki, "Whispering-gallery-mode-resonator-based ultranarrow linewidth external-cavity semiconductor laser," Opt. Lett., vol. 35, no. 16, pp. 2822-2824, 2010.

[47] A. Jiménez, T. Milde, N. Staacke, C. Assmann, G. Carpintero, and J. Sacher, "Narrow-line external cavity diode laser micropackaging in the nir and mir spectral range," Applied Physics B, vol. 123, no. 7, p. 207, 2017.
[48] J. O'Carroll, R. Phelan, B. Kelly, D. Byrne, L. P. Barry, and J. O'Gorman, "Wide temperature range $0<t<85^{\circ} \mathrm{c}$ narrow linewidth discrete mode laser diodes for coherent communications applications," Opt. Express, vol. 19, no. 26, pp. B90-B95, 2011.

[49] L. Richter, H. Mandelberg, M. Kruger, and P. McGrath, "Linewidth determination from self-heterodyne measurements with subcoherence delay times," IEEE J. Quantum Electron., vol. 22, no. 11, pp. 2070-2074, 1986.

[50] D. S. Wu, R. Slavík, G. Marra, and D. J. Richardson, "Direct selection and amplification of individual narrowly spaced optical comb modes via injection locking: design and characterization," J. Lightw. Technol., vol. 31, no. 14, pp. 2287-2295, 2013.

[51] R. Kakarla, J. Schröder, and P. A. Andrekson, "Optical injection locking at sub nano-watt powers," Opt. Lett., vol. 43, no. 23, pp. 5769-5772, 2018.

[52] G. Bosco, V. Curri, A. Carena, P. Poggiolini, and F. Forghieri, "On the performance of nyquist-wdm terabit superchannels based on pm-bpsk, pm-qpsk, pm-8qam or pm-16qam subcarriers," J. Lightw. Technol., vol. 29, no. 1, pp. 53-61, 2010.

[53] S. T. Cundiff and A. M. Weiner, "Optical arbitrary waveform generation," Nat. Photonics, vol. 4, no. 11, p. 760, 2010.

[54] R. Scott, N. Fontaine, J. Cao, K. Okamoto, B. Kolner, J. Heritage, and S. B. Yoo, "High-fidelity line-by-line optical waveform generation and complete characterization using frog," Opt. Express, vol. 15, no. 16, pp. 9977-9988, 2007.

[55] T. M. Fortier, M. S. Kirchner, F. Quinlan, J. Taylor, J. Bergquist, T. Rosenband, N. Lemke, A. Ludlow, Y. Jiang, C. Oates et al., "Generation of ultrastable microwaves via optical frequency division," Nat. Photonics, vol. 5, no. 7, p. 425, 2011.

[56] P. Marin-Palomo, J. N. Kemal, M. Karpov, A. Kordts, J. Pfeifle, M. H. Pfeiffer, P. Trocha, S. Wolf, V. Brasch, M. H. Anderson et al., "Microresonator-based solitons for massively parallel coherent optical communications," Nature, vol. 546, no. 7657, p. 274, 2017.

[57] Z. Wang, K. Van Gasse, V. Moskalenko, S. Latkowski, E. Bente, B. Kuyken, and G. Roelkens, "A iii-v-on-si ultra-dense comb laser," Light: Science \& Applications, vol. 6, no. 5, p. e16260, 2017.

[58] G. P. Agrawal, Fiber-optic communication systems. John Wiley \& Sons, 2012, vol. 222

[59] K. Kobayashi, R. Lang, and K. Minemura, "Novel optical methods for high speed direct modulation of semiconductor lasers," Japanese Journal of Applied Physics, vol. 15, no. S1, p. $281,1976$.

[60] K. Balakier, L. Ponnampalam, M. J. Fice, C. C. Renaud, and A. J. Seeds, "Integrated semiconductor laser optical phase lock loops," IEEE J. Sel. Topics Quantum Electron., vol. 24, no. 1, pp. 1-12, 2017.

[61] J. Kim, H. Schnatz, D. S. Wu, G. Marra, D. J. Richardson, and R. Slavík, "Optical injection locking-based amplification in phase-coherent transfer of optical frequencies," Opt. Lett., vol. 40, no. 18, pp. 4198-4201, 2015.

[62] Z. Liu, J.-Y. Kim, D. S. Wu, D. J. Richardson, and R. Slavik, "Homodyne ofdm with optical injection locking for carrier recovery," J. Lightw. Technol., vol. 33, no. 1, pp. 34-41, 2014.

[63] S. Harris, B. McMurtry, and A. Siegman, "Modulation and direct demodulation of coherent and incoherent light at a microwave frequency," Applied Physics Letters, vol. 1, no. 2, pp. 37-39, 1962.

[64] Y. Matsui, W. Li, H. Roberts, H. Bulthuis, H. Deng, L. Lin, and C. Roxlo, "Transceiver for ng-pon2: Wavelength tunablity for burst mode twdm and point-to-point wdm," in Optical Fiber Communications. IEEE, 2016, pp. 1-3.

[65] F. Chang, Datacenter connectivity technologies: principles and practice. River Publishers, Gistrup, 2019.

[66] J. Bowers, D. Liang, A. Fang, H. Park, R. Jones, and M. Paniccia, "Hybrid silicon lasers: the final frontier to integrated 
computing," Optics and Photonics News, vol. 21, no. 5, pp. 28-33, 2010.

[67] Z. Liu, B. Kelly, J. O'Carroll, R. Phelan, D. J. Richardson, and R. Slavík, "Discrete multitone format for repeater-less directmodulation direct-detection over $150 \mathrm{~km}$," J. Lightw. Technol., vol. 34, no. 13, pp. 3223-3229, 2016.

[68] U. Troppenz, J. Kreissl, M. Möhrle, C. Bornholdt, W. Rehbein, B. Sartorius, I. Woods, and M. Schell, " $40 \mathrm{gbit} / \mathrm{s}$ directly modulated lasers: Physics and application," in SPIE, vol. 7953, 2011, p. 1-10.

[69] Y. Matsui, R. Schatz, T. Pham, W. A. Ling, G. Carey, H. M. Daghighian, D. Adams, T. Sudo, and C. Roxlo, "55 ghz bandwidth distributed reflector laser," J. Lightw. Technol., vol. 35, no. 3, pp. 397-403, 2017.

[70] X. Zhao, D. Parekh, E. K. Lau, H.-K. Sung, M. C. Wu, W. Hofmann, M. C. Amann, and C. J. Chang-Hasnain, "Novel cascaded injection-locked $1.55-\mu \mathrm{m}$ vcsels with $66 \mathrm{ghz}$ modulation bandwidth," Opt. Express, vol. 15, no. 22, pp. 14810 14 816, 2007.

[71] Y.-C. Chi, Y.-C. Li, H.-Y. Wang, P.-C. Peng, H.-H. Lu, and G.-R. Lin, "Optical 16-qam-52-ofdm transmission at $4 \mathrm{gbit} / \mathrm{s}$ by directly modulating a coherently injection-locked colorless laser diode," Opt. Express, vol. 20, no. 18, pp. 20071-20 077, 2012.

[72] Z. Liu, Y. Chen, Z. Li, B. Kelly, R. Phelan, J. O'Carroll, T. Bradley, J. P. Wooler, N. V. Wheeler, A. M. Heidt et al., "High-capacity directly modulated optical transmitter for $\overline{2-\mu \mathrm{m}}$ spectral region,” J. Lightw. Technol., vol. 33, no. 7, pp. 13731379, 2015.

[73] R. Slavik, J. Kakande, R. Phelan, J. O'Carroll, B. Kelly, and D. Richardson, "Wavelength-tunable qam synthesis by direct modulation of injection-locked fabry-perot semiconductor lasers," in Optical Fiber Communication Conference. IEEE, 2013, pp. 1-3.

[74] H.-H. Lu, C.-Y. Li, H.-W. Chen, Z.-Y. Yang, X.-Y. Lin, M.-T. Cheng, C.-K. Lu, and T.-T. Shih, " $45 \mathrm{gb} / \mathrm{s}$ pam4 transmission based on vcsel with light injection and optoelectronic feedback techniques," Opt. Lett., vol. 41, no. 21, pp. 5023-5026, 2016.

[75] H.-H. Lu, C.-Y. Li, H.-W. Chen, C.-M. Ho, M.-T. Cheng, Z.-Y. Yang, and C.-K. Lu, "A $56 \mathrm{gb} / \mathrm{s}$ pam4 vcsel-based lifi transmission with two-stage injection-locked technique," IEEE Photonics Journal, vol. 9, no. 1, pp. 1-8, 2016.

[76] D. Liang, Z. Liu, A. Descos, S. Srinivasan, Z. Huang, Kurczveil, Geza, and G. R. Beausoleil, "Optical injection-locked high-speed heterogeneous quantum-dot microring lasers," in European Conference on Optical Communication, 2019, p. Th.2.D.6.

[77] Z. Liu, J. Kakande, B. Kelly, J. O'Carroll, R. Phelan, D. J. Richardson, and R. Slavík, "Modulator-free quadrature amplitude modulation signal synthesis," Nat. Commun., vol. 5, p. $5911,2014$.

[78] Z. Liu, G. Hesketh, B. Kelly, J. O'Carroll, R. Phelan, D. J. Richardson, and R. Slavík, "Optical injection-locked directly modulated lasers for dispersion pre-compensated directdetection transmission," J. Lightw. Technol., vol. 36, no. 20, pp. 4967-4974, 2018.

[79] X. Xiao, N. K. Fontaine, H. Chen, B. Huang, D. T. Neilson, K. Kim, J. H. Sinsky, R. R. Ryf, G. Raybon, P. Winzer et al., "High-speed iq modulator based on injection-locked vcsel array," in CLEO: Science and Innovations. Optical Society of America, 2017, pp. STu1M-6.

[80] N. K. Fontaine, X. Xiao, H. Chen, B. Huang, D. T. Neilson, K. Kim, J. H. Sinsky, R. R. Ryf, G. Raybon, P. Winzer et al., "Chirp-free modulator using injection locked vcsel phase array," in European Conference on Optical Communication. VDE, 2016, pp. 1-3.

[81] C. Wang, M. Zhang, X. Chen, M. Bertrand, A. Shams-Ansari, S. Chandrasekhar, P. Winzer, and M. Lončar, "Integrated lithium niobate electro-optic modulators operating at cmos-compatible voltages," Nature, vol. 562, no. 7725, p. 101, 2018.

[82] A. Albores-Mejia, T. Kaneko, E. Banno, K. Uesaka, H. Shoji, and $\mathrm{H}$. Kuwatsuka, "Optical-comb-line selection from a lowpower/low-osnr comb using a low-coherence semiconductor laser for flexible ultra-dense short range transceivers," in Optical Fiber Communication Conference. Optical Society of America, 2015, pp. W2A-23.

[83] Z. Liu, S. Farwell, M. Wale, D. J. Richardson, and R. Slavík, "Inp-based optical comb-locked tunable transmitter," in Optical Fiber Communication Conference. Optical Society of America, 2016, pp. Tu2K-2.

[84] Z. Liu, S. G. Farwell, M. J. Wale, D. J. Richardson, and R. Slavík, "Inp-based comb-locked optical super channel transmitter," in Asia Communications and Photonics Conference. Optical Society of America, 2016, pp. AS2C-1.

[85] R. Zhou, T. Shao, M. D. G. Pascual, F. Smyth, and L. P. Barry, "Injection locked wavelength de-multiplexer for optical comb-based nyquist wdm system," IEEE Photon. Technol. Lett., vol. 27, no. 24, pp. 2595-2598, 2015.

[86] E. Temprana, E. Myslivets, B. P.-P. Kuo, N. Alic, and S. Radic, "Transmitter-side digital back propagation with optical injection-locked frequency referenced carriers," J. Lightw. Technol., vol. 34, no. 15, pp. 3544-3549, 2016.

[87] Y. Wang, K. Kasai, M. Yoshida, and M. Nakazawa, "320 gbit/s, 20 gsymbol/s 256 qam coherent transmission over $160 \mathrm{~km}$ by using injection-locked local oscillator," Opt. Express, vol. 24, no. 19, pp. $22088-22096,2016$.

[88] M. Mazur, J. Schröder, A. Lorences-Riesgo, T. Yoshida, M. Karlsson, and P. A. Andrekson, " $12 \mathrm{~b} / \mathrm{s} / \mathrm{hz}$ spectral efficiency over the c-band based on comb-based superchannels," J. Lightw. Technol., vol. 37, no. 2, pp. 411-417, 2018.

[89] J. Jignesh, B. Corcoran, J. Schröder, and A. Lowery, "Polarization independent optical injection locking for carrier recovery in optical communication systems," Opt. Express, vol. 25, no. 18, pp. $21216-21228,2017$.

[90] A. J. Seeds, M. J. Fice, K. Balakier, M. Natrella, O. Mitrofanov, M. Lamponi, M. Chtioui, F. van Dijk, M. Pepper, G. Aeppli et al., "Coherent terahertz photonics," Opt. Express, vol. 21, no. 19 , pp. $22988-23000,2013$.

[91] E. Temprana, E. Myslivets, B.-P. Kuo, L. Liu, V. Ataie, N. Alic, and S. Radic, "Overcoming kerr-induced capacity limit in optical fiber transmission," Science, vol. 348, no. 6242, pp. $1445-1448,2015$.

[92] S. Xiao, L. Hollberg, N. R. Newbury, and S. A. Diddams, "Toward a low-jitter $10 \mathrm{ghz}$ pulsed source with an optical frequency comb generator," Opt. Express, vol. 16, no. 12, pp. 8498-8508, 2008.

[93] J. Pfeifle, V. Brasch, M. Lauermann, Y. Yu, D. Wegner, T. Herr, K. Hartinger, P. Schindler, J. Li, D. Hillerkuss et al., "Coherent terabit communications with microresonator kerr frequency combs," Nat. Photonics, vol. 8, no. 5, p. 375, 2014.

[94] H. Hu, F. Da Ros, M. Pu, F. Ye, K. Ingerslev, E. P. da Silva, M. Nooruzzaman, Y. Amma, Y. Sasaki, T. Mizuno et al., "Single-source chip-based frequency comb enabling extreme parallel data transmission," Nat. Photonics, vol. 12, no. 8, p. 469, 2018

[95] D. J. Geisler, N. K. Fontaine, R. P. Scott, T. He, L. Paraschis, O. Gerstel, J. P. Heritage, and S. Yoo, "Bandwidth scalable, coherent transmitter based on the parallel synthesis of multiple spectral slices using optical arbitrary waveform generation," Opt. Express, vol. 19, no. 9, pp. 8242-8253, 2011.

[96] R. Slavík, S. G. Farwell, M. J. Wale, and D. J. Richardson, "Compact optical comb generator using inp tunable laser and push-pull modulator," IEEE Photon. Technol. Lett., vol. 27, no. 2, pp. 217-220, 2014.

[97] P. J. Winzer, C. J. Chang-Hasnain, A. E. Willner, R. C. Alferness, R. W. Tkach, and T. G. Giallorenzi, "A third of a century 
of lightwave technology january 1983-april 2016," J. Lightw. Technol., vol. 34, no. 9, pp. 2079-2084, 2016.

[98] A. Tartaglia, "Optics for 5g: How can we combine low cost with demanding requirements?" in European Conference on Optical Communication. VDE, 2016, pp. 1-3.

[99] J. K. Perin, A. Shastri, and J. M. Kahn, "Design of low-power dsp-free coherent receivers for data center links," J. Lightw. Technol., vol. 35, no. 21, pp. 4650-4662, 2017.

[100] P. Minzioni, C. Lacava, T. Tanabe, J. Dong, X. Hu, G. Csaba, W. Porod, G. Singh, A. E. Willner, A. Almaiman, V. TorresCompany, J. Schröder, A. C. Peacock, M. J. Strain, F. Parmigiani, G. Contestabile, D. Marpaung, Z. Liu, J. E. Bowers, L. Chang, S. Fabbri, M. R. Vázquez, V. Bharadwaj, S. M. Eaton, P. Lodahl, X. Zhang, B. J. Eggleton, W. J. Munro, K. Nemoto, O. Morin, J. Laurat, and J. Nunn, "Roadmap on all-optical processing," J. of Optics, vol. 21, no. 6, p. 063001, may 2019.

[101] M. J. Fice, A. Chiuchiarelli, E. Ciaramella, and A. J. Seeds, "Homodyne coherent optical receiver using an optical injection phase-lock loop," J. Lightw. Technol., vol. 29, no. 8, pp. 1152$1164,2011$.

[102] A. Chiuchiarelli, M. J. Fice, E. Ciaramella, and A. J. Seeds, "Effective homodyne optical phase locking to psk signal by means of 8b10b line coding," Opt. Express, vol. 19, no. 3, pp. 1707-1712, 2011.

[103] S. L. Jansen, I. Morita, T. C. Schenk, N. Takeda, and H. Tanaka, "Coherent optical $25.8 \mathrm{-gb} / \mathrm{s}$ ofdm transmission over $4160-\mathrm{km}$ ssmf," J. Lightw. Technol., vol. 26, no. 1, pp. 6-15, 2008.

[104] B. Inan, S. Randel, S. L. Jansen, A. Lobato, S. Adhikari, and N. Hanik, "Pilot-tone-based nonlinearity compensation for optical ofdm systems," in European Conference on Optical Communication. IEEE, 2010, pp. 1-3.

[105] S. Adhikari, S. Sygletos, A. D. Ellis, B. Inan, S. L. Jansen, and W. Rosenkranz, "Enhanced self-coherent ofdm by the use of injection locked laser," in Optical Fiber Communication Conference. Optical Society of America, 2012, pp. JW2A64.

[106] M. Mazur, A. Lorences-Riesgo, J. Schröder, P. A. Andrekson, and M. Karlsson, "10 tb/s pm-64qam self-homodyne combbased superchannel transmission with $4 \%$ shared pilot tone overhead," J. Lightw. Technol., vol. 36, no. 16, pp. 3176-3184, 2018.

[107] V. Torres-Company, J. Schroeder, A. Fülöp, M. Mazur, L. Lundberg, O. Helgason, M. Karlsson, and P. Andrekson, "Laser frequency combs for coherent optical communications," J. Lightw. Technol., 2019.

[108] H. Wernz, S. Bayer, B.-E. Olsson, M. Camera, H. Griesser, C. Furst, B. Koch, V. Mirvoda, A. Hidayat, and R. Noé, "112gb/s polmux rz-dqpsk with fast polarization tracking based on interference control," in Optical Fiber Communication Conference. IEEE, 2009, pp. 1-3.

[109] J. Jignesh, B. Corcoran, C. Zhu, and A. Lowery, "Simple optoelectronic frequency-offset estimator for coherent optical ofdm," Opt. Express, vol. 25, no. 25, pp. 32 161-32 177, 2017.

[110] K. Croussore and G. Li, "Phase regeneration of nrz-dpsk signals based on symmetric-pump phase-sensitive amplification," IEEE Photon. Technol. Lett., vol. 19, no. 11, pp. 864-866, 2007.

[111] R. Slavík, F. Parmigiani, J. Kakande, C. Lundström, M. Sjödin, P. A. Andrekson, R. Weerasuriya, S. Sygletos, A. D. Ellis, L. Grüner-Nielsen, D. Jakobsen, S. Herstrøm, R. Phelan, J. O'Gorman, A. Bogris, D. Syvridis, S. Dasgupta, P. Petropoulos, and D. J. Richardson, "All-optical phase and amplitude regenerator for next-generation telecommunications systems," Nat. Photonics, vol. 4, no. 10, p. 690, 2010.

[112] J. Kakande, A. Bogris, R. Slavík, F. Parmigiani, D. Syvridis, P. Petropoulos, D. Richardson, M. Westlund, and M. Sköld, "Qpsk phase and amplitude regeneration at 56 gbaud in a novel idler-free non-degenerate phase sensitive amplifier," in Optical Fiber Communication Conference. Optical Society of America, 2011, p. OMT4.
[113] J. Kakande, R. Slavík, F. Parmigiani, A. Bogris, D. Syvridis, L. Grüner-Nielsen, R. Phelan, P. Petropoulos, and D. J. Richardson, "Multilevel quantization of optical phase in a novel coherent parametric mixer architecture," Nat. Photonics, vol. 5, no. 12, p. 748, 2011.

[114] Z. Tong, C. Lundström, M. Karlsson, M. Vasilyev, and P. Andrekson, "Noise performance of a frequency nondegenerate phase-sensitive amplifier with unequalized inputs," Opt. Lett., vol. 36, no. 5, pp. 722-724, 2011.

[115] R. Kakarla, J. Schröder, and P. A. Andrekson, "Recordsensitivity $\mathrm{gb} / \mathrm{s}$ receiver for free-space applications based on phase-sensitive amplification," in CLEO: Applications and Technology. Optical Society of America, 2019, pp. JTh5B-1.

[116] R. Slavík, J. Kakande, and D. J. Richardson, "Feed-forward true carrier extraction of high baud rate phase shift keyed signals using photonic modulation stripping and low-bandwidth electronics," Opt. Express, vol. 19, no. 27, pp. 26 594-26599, 2011.

[117] R. Slavík, F. Parmigiani, L. Gruner-Nielsen, D. Jakobsen, S. Herstrom, P. Petropoulos, and D. Richardson, "Stable and efficient generation of high repetition rate $(>160 \mathrm{ghz})$ subpicosecond optical pulses," IEEE Photon. Technol. Lett., vol. 23, no. 9, pp. 540-542, 2011.

[118] B. P.-P. Kuo, E. Myslivets, V. Ataie, E. G. Temprana, N. Alic, and S. Radic, "Wideband parametric frequency comb as coherent optical carrier," J. Lightw. Technol., vol. 31, no. 21, pp. 3414-3419, 2013.

[119] V. Ataie, D. Esman, B.-P. Kuo, N. Alic, and S. Radic, "Subnoise detection of a fast random event," Science, vol. 350, no. 6266, pp. 1343-1346, 2015

[120] E. Temprana, V. Ataie, B. P.-P. Kuo, E. Myslivets, N. Alic, and S. Radic, "Low-noise parametric frequency comb for continuous c-plus-1-band 16-qam channels generation," Opt. Express, vol. 22, no. 6, pp. 6822-6828, 2014.

[121] A. M. Weiner, "Femtosecond pulse shaping using spatial light modulators," Review of Scientific Instruments, vol. 71, no. 5, pp. 1929-1960, 2000.

[122] Z. Jiang, D. S. Seo, D. E. Leaird, and A. M. Weiner, "Spectral line-by-line pulse shaping," Opt. Lett., vol. 30, no. 12, pp. 1557$1559,2005$.

[123] G. J. Schnneider, J. A. Murakowski, C. A. Schuetz, S. Shi, and D. W. Prather, "Radiofrequency signal-generation system with over seven octaves of continuous tuning," Nat. Photonics, vol. 7, pp. 118-122, 2013.

[124] N. Hinkley, J. Sherman, N. Phillips, M. Schioppo, N. Lemke, K. Beloy, M. Pizzocaro, C. W. Oates, and A. Ludlow, "An atomic clock with 10-18 instability," Science, vol. 341, no. 6151, pp. 1215-1218, 2013.

[125] S. Droste, F. Ozimek, T. Udem, K. Predehl, T. Hänsch, H. Schnatz, G. Grosche, and R. Holzwarth, "Optical-frequency transfer over a single-span $1840 \mathrm{~km}$ fiber link," Physical Review Letters, vol. 111, no. 11, p. 110801, 2013.

[126] J. Kim, G. Marra, D. S. Wu, D. J. Richardson, and R. Slavík, "Wavelength conversion technique for optical frequency dissemination applications," Opt. Lett., vol. 41, no. 8, pp. 1716-1719, 2016.

[127] H. Wang, D. Kim, M. Harfouche, C. T. Santis, N. Satyan, G. Rakuljic, and A. Yariv, "Narrow-linewidth oxide-confined heterogeneously integrated si/iii-v semiconductor lasers," IEEE Photon. Technol. Lett., vol. 29, no. 24, pp. 2199-2202, 2017.

[128] A. Y. Liu, T. Komljenovic, M. L. Davenport, A. C. Gossard, and J. E. Bowers, "Reflection sensitivity of $1.3 \mu \mathrm{m}$ quantum dot lasers epitaxially grown on silicon," Opt. Express, vol. 25, no. 9, pp. 9535-9543, 2017. 